\title{
Synergistic Inhibitory Effect of lonizing Radiation Combined With Imatinib on Gastrointestinal Stromal Tumor Cell and Its Mechanism
}

\section{Wenbiao Xiao ( $\nabla$ xiaowenbiao1@163.com )}

The first affiliated hospital of Fujian medical university

\section{Yongjian Zhou}

Xiehe Affiliated Hospital of Fujian Medical University

Guosheng Lin

Xiehe Affiliated Hospital of Fujian Medical University

Renjie Guo

Xiehe Affiliated Hospital of Fujian Medical University

\section{Research}

Keywords: Imatinib, ionizing radiation, gastrointestinal stromal tumor, synergistic inhibition, Rad51

Posted Date: November 12th, 2020

DOI: https://doi.org/10.21203/rs.3.rs-103663/v1

License: (c) (i) This work is licensed under a Creative Commons Attribution 4.0 International License. Read Full License 


\section{Abstract}

\section{Background}

RT is considered insensitive to GIST,However, recently, the effective clinical practice of RT in GIST has been reported,The purpose of this study was to clarify the synergistic effect of RT combined with IM on GIST and the potential molecular mechanism.

\section{Methods}

The expression of DNA repair genes related to IR in 100 patients with GIST and their matched tissues was studied by IHC.The relationship between them and clinicopathological characteristics and risk of GIST was analyzed.The effects of IR combined with IM on the proliferation, clonogenesis, invasion and migration, cell cycle and apoptosis of GIST-882 cell were examined by cell count, CCK-8, plate cloning, scratch and transwell, and flow cytometry.Combined with KEGG database signal pathway analysis, qPCR and westernblot were used to study the potential molecular mechanism of coordinated inhibition of IM combined with IR on GIST-882 cell.

\section{Results}

The expression of DNA repair protein related to IR injury in GIST was significantly higher than that in matched adjacent tissues, and the high expression of Rad51 was related to the high risk of GIST ( $\mathrm{P}<$ 0.05).The interaction between IR and IM was strong by factorial design analysis $(P<0.05)$. The combined index of choutalalay analysis also proved the above conclusion.Compared with the single effect, the combined effect significantly enhanced the ability of clone formation, migration and invasion, and the inhibition of apoptosis of GIST-882 cells.In cell cycle study,IM mainly leads to direct apoptosis, while IR leads to cell cycle arrest in S and G2 / M phases.In the study of molecular mechanism, it was found that IR damage can feedback stimulate the high expression of DNA repair protein in cells, while im may enhance IR sensitivity by inhibiting the expression of radiation damage related DNA repair protein Rad51 in p53 signaling pathway.

\section{Conclusions}

IM combined with IR can synergistically inhibit the proliferation, apoptosis, clonogenesis, invasion, migration and cell cycle of GIST cells. The mechanism of synergism may be through IM to inhibit p53, a signal pathway of radiation damage repair.

\section{Background}

Gastrointestinal stromal tumors (GIST) are the most commonmesenchymal tumors in the gastrointestinal tract, with an annual incidence of about 1-3/0.1 million(1)Mesylate Imatinib(IM) is a firstline drug for the treatment of advanced GIST $\llbracket 2-4$ ). However, $70 \%-80 \%$ of patients have secondary drug resistance, which seriously affects the prognosis(5-8). In the past, it was generally believed that the 
radiosensitivity of GIST was lacking,only the palliative treatment of GIST bonemetastasis was recommended in the guidelines(9),it is mainly due to the slow response rate of RT in the treatment of soft tissue sarcoma, and the early retrospectivecase study has little benefit.Secondly, the limitations of imaging and radiotherapy (RT) technology make it difficult for GIST in the abdominal cavity toreceive local high-dose radiation $\otimes 10,11 \rrbracket$.

With the development of tumor molecular heterogeneity theory(12), there are still some patients with GIST who are sensitive to radiotherapy, especially for the patients with advanced stage(13-16).In addition, with the development of imaging technology and modern tissue and organ radiation technology, it has become a reality to concentrate high-dose radiation locally in abdominal cavity tumors(12), which challenges RT's insensitivity to GISTs. In recent years, reports on the efficacy and safety of RT in GIST have been emerging (17-21),the RT research of 15 cases of GIST published by John J Cuaron (22) in 2013 and the RT single arm clinical trial of 25 cases of GIST published by Heikki Joensuu (23) in 2015 provide the effective basis for the application of RT in GIST.

Coincidentally, we looked for RT in GIST and found that IM plays a role in radiosensitization in many other tumors(24-27), but the internal molecular mechanism is not unified $(28,29)$.Some studies have suggested that it may be closely related to the homologous recombination (HR) repair protein Rad51 (30), IM can cause the translation of Rad51 to be blocked(31-33). But the basic research of RT combined IM in GIST has never been reported. Therefore, this study studies the synergistic inhibition of IM combined with ionizing radiation(IR) on the biological behavior of GIST-882 at the cellular level, which provides a new treatment idea for the current targeted treatment of GIST.

\section{Methods}

\section{Human tissues}

The pathological sections of 100 cases of gastrointestinal stromal tumors and their corresponding paratumoral tissues (at least $5 \mathrm{~cm}$ away from the tumor) were provided from Xiehe Hospital Affiliated to Fujian Medical University,the clinicopathological data of these patients were collected in detail.All patients had not received any radiotherapy, chemotherapy and targeted treatment, and received gastrointestinal stromal tumor resection.The samples of 100 patients (2010-2018 gastrectomy) were collected for immunohistochemical staining. This study was approved by the ethics committee of Union Hospital of Fujian Medical University and obtained the written consent of all patients.

Main instruments

Flow cytometry: BD company, USA; RS2000: source;

Immunohistochemistry $(\mathrm{IHC})$

Immunohistochemistry (IHC) analysis have previously been described as [15]. See attachment 1 for details. 
Human gist cell line (GIST-882 cell) was purchased from cosmobio company in Japan. The cells were cultured in 1640 (GIBCO, Grand Island, NY) or DMEM medium containing 10\% 15\% fetal bovine serum at $37^{\circ} \mathrm{C}$ and $5 \% \mathrm{CO} 2$.

IM concentration preparation

The powder im (Sigma: sml1027) was first prepared into $10 \mathrm{mmol} / \mathrm{L}$ stock solution, and then diluted according to the required concentration;

Cell proliferation experiment

Cell morphology observation and cell counting method: the cells in the logarithmic growth period were intervened by different factors. After $48 \mathrm{~h}$, the changes of cell morphology were observed under the inverted microscope and the cell viability was counted. The cell proliferation experiment was carried out according to the instructions of CCK8 KIT, and the details are shown in Appendix 1;

Plate cell clonogenesis experiment

The GIST-882 cell lines of different intervention groups were made into cell suspension and diluted with gradient multiple. The cell density of 8000 cells/well was respectively inoculated into the six pore plate, three of them were re porous, mixed and placed in the incubator. Regular observation, when there are visible clones, terminate the culture. Discard supernatant and wash twice. Fixed with $4 \%$ paraformaldehyde, add appropriate amount of Gimsa for dyeing, wash off the dyeing solution slowly with running water and dry. Put the plate upside down and add a piece of transparent film with grid, and count more than 50 cell clones under microscope (low power microscope);

Migration and invasion experiments

GIST-882 cells were treated with IM at different concentrations for 24 hours and then treated with IR at different doses. The distance of cell migration was observed and recorded regularly. 72 hours later, cell migration around the scratch was observed under inverted microscope. The lateral migration area of each treatment group was calculated by ImageJ software. Transwell migration and invasion experiment: $5 \times$ 104 gist cells (200ul cell suspension) in each Transwell cell chamber (with or without matrix glue), 800ul complete culture medium containing $20 \%$ FBS was placed at the bottom of the chamber, and the matrix and cells in the upper chamber were wiped with cotton swabs 48 hours later, and the cells were counted under the microscope after crystal violet staining.

Detection of early apoptosis

Set the cell density to $5 \times 105 / \mathrm{ml}$ (inoculated on the six pore plate), set it to 4 groups, 3 multiple pores, respectively as the control group, im2um, ir100gy, IM + IR; after 24 hours of wall sticking, Im intervention, and IR after 24 hours; after 24 hours of IR, digestion and washing of cells: count the cells, so that the 
number of cells in each tube is between $5 \times 105-1 \times 106$, add 500ul $1 \times$ bindingbuffer suspension cells according to the instructions of the kit, etc Operation, flow cytometry detection.

Detection of cell cycle and late apoptosis

The treatment was the same as that of the control group, $I M=0.5 u M, I M=2 u M, I R=100 G y, I R=150 G y$, $I M=0.5 u M+I R=150 G y, I M=2 u M+I R=100 G y$; the treatment was the same as that of the control group, centrifuged and fixed with $70 \%$ ethanol, $4{ }^{\circ} \mathrm{C}$ overnight. Pre cooled PBS, re suspension cells, centrifuged cells; propidium iodide staining solution, after avoiding light and temperature bath, re suspension cells with PBS, flow cytometry detection and analysis.

Quantitativereal-timePCR

Sample collection and RNA extraction and reverse transcription:The above-mentioned treatment method involves cell 6 intervention under different conditions, PBS washing once, RNA extraction according to the instructions, quantitative RNA concentration and purity, and reverse transcription to CDNA at $-80{ }^{\circ} \mathrm{C}$; the primer sequence of the target gene is obtained by referring to relevant literature, as follows:

ATM:(F:TGGATCCAGCTATTTGGTTTGA,R:CCAAGTATGTAACCAACAACAATAGAAGAAG)

P53: (F:TGGTTCCATCAGTCAGGTCA, R:ACAGCAGGAGCAGATTCCAC)『

RAD51:(F:CAGATGCAGCTTGAAGCAAA, R: TTCTTCACATCGTTGGCATT)』

GAPDH:(F:TGGTTCCATCAGTCAGGTCA, R: ACAGCAGGAGCAGATTCCAC)『

Real time fluorescence quantification:Using Promega GoScript Taq qPCR Master Mi kit,the reaction system was constructed by the kit, each reaction was set up with 3 repetitive systems, and the relative expression was calculated by the software;

Western Blot assay

Western blot analysis was performed using the following antibodies $\triangle A T M / A T R(\# 2851) ; r-$ H2A.X(ab2893);P53(\#2527);RAD51(ab133534);Ku70+Ku80(ab53126);AKT(\#4685);pAKT(\#4080);Erk1/2(\#4695);p-Erk1/2(\#4370); $\beta$-actin(\#4970);See attachment 1 for details.

Statistical methods

All the data were processed using the SPSS23.0 statistical software package and Prism 7.0 software (GraphPad).Continuous values are expressed as the mean \pm standard deviation and analyzed using the Student's $t$ test.Categorical variables were analyzed using the $\chi 2$ or Fisher's exact tests. The synergistic effect of the two factors was analyzed by factorial analysis and repeated analysis. The difference was considered statistically significant at $\mathrm{P}<0.05$. 


\section{Results}

\section{Radiation-related DNA damage repair protein are increased in GIST}

Immunohistochemical staining was used,the expression rates of GIST in 80 cases (Rad51), 50 cases (yH2A.X), 30 cases (Ku70/80) and their corresponding adjacent tissues were $93.75 \%$ vs $43.75 \%, 80.00 \%$ vs $26.00 \%$ and $43.33 \%$ vs $10.00 \%$, respectively. The differences were statistically significant (Figure 1A, B and C). The high expression of Rad51 protein was positively correlated with the high risk of GIST ( $<<$ 0.05 ) (Table 1).It is suggested that the radiation-related DNA damage repair protein is closely related to the genesis and development of GIST.

\section{IR combined with IM significantly inhibited the biological behavior of GIST-882 cell}

In order to study the combined effect, we selected IM=1uM, $2 \mathrm{uM}$ and IR=100Gy to observe under the microscope respectively. It was found that compared with each single group, the cell density of the combined group decreased significantly, and the cells gradually shrank and grew, and the state was gradually disintegrating $\triangle$ Figure $2 \mathrm{~A} \triangle \mathrm{B} \otimes$.The number of cells in the combined group decreased significantly(Figure 2 C).CCK8 method was used to detect the inhibitory effect of IM and IR on cell growth,the IC50 (EC50) of IM was 7.775uM, 4.068uM and 1.865uM at 24h, 48h and 72h, and IR was 231.78Gy, 123.80Gy and 417.80Gy, respectively.Repeated measurement analysis of variance showed that GIST-882 cells had time-dependent( $P 1<0.01, P 2<0.01)$ and dose-dependent $(\mathrm{P} 1<0.01 ; \mathrm{P} 2<0.01)$ effects on IM and IR, and IM concentration and time had synergistic effect $(P<0.01)$ (Figure3A,B).

The results of factorial analysis showed that IM and IR had a strong interaction $(P<0.01)$, indicating that

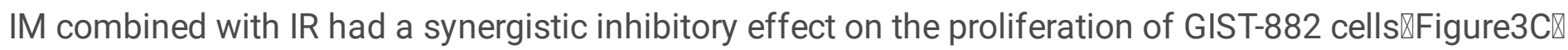

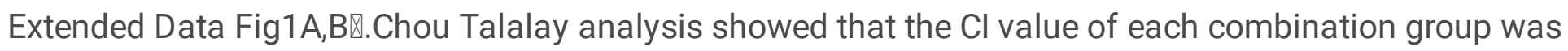
less than 0.7 , that is, there is at least slight synergy, and the $\mathrm{Cl}$ of multiple combination groups was less than 0.3 , showing strong synergistic effect(Figure 3D, table 2).By analyzing the effect of IM on EC50 in IR group, we found that EC50 in IR group was 231.78Gy without IM treatment, while EC50 in IR group dropped to 37.85Gy when IM = 1uM®Figure 3E囚.

Compared with the control group, the number of cell clones in $\mathrm{IM}=1 \mathrm{uM}$ group, $\mathrm{IR}=100 \mathrm{~Gy}$ group and combination group decreased significantly, and the difference was statistically significant.But compared with the IM=1 $\mathrm{uM}$ group alone, the cell clone number of the combined group was lower. Although there was no significant difference between the $\mathrm{IR}=100 \mathrm{~Gy}$ group and the combined group, cell colonies had been found in the IR group, but there was no cell colony formation in the combined groupهFigure3F,3G).

In terms of cell migration and invasion ability, the combined group had more significant inhibition on cell lateral migration ability (P1 < 0.01, P1 < 0.01) (Figure 4A), and the Transwell chamber vertical migration experiment showed the same result (Figure 4B). The invasion experiment showed that compared with IM and IR alone, the number of invasive cells in the combined group decreased significantly, and the difference was statistically significant $(P 1<0.01, \mathrm{P} 2<0.01)$ (Figure $4 \mathrm{C}$ ). 


\section{Combined action inhibited the early apoptosis, cell cycle and late apoptosis of GIST-882 cell}

In the aspect of early apoptosis, $I M=2 \mathrm{uM}, \mathrm{IR}=100 \mathrm{G}$ and the combination group all increased the apoptosis of GIST-882 cells, and the difference was statistically significant (P1 $<0.05, \mathrm{P} 2<0.05, \mathrm{P} 3<$ 0.01). However, compared with the group of $\mathrm{IM}=2 \mathrm{uM}$, there was no significant difference in the apoptosis rate of the combined group $(P=0.102)$, compared with the group of $I R=100 \mathrm{~Gy}(P 1<0.05)$ (Figure $5 A)$.

Because G0/G1 phase indicates late apoptosis, G2/M phase indicates that cells will enter the pre-mitotic stage, and cell accumulation represents cell cycle arrest, so we focused on analyzing the effects of different intervention groups in these two stages.

In terms of late apoptosis, in G0/G1 phase: compared with IM group, the late apoptosis rate of combined group was significantly increased, and IR could enhance the late apoptosis effect of IM on cells (Figure $5 B)$.Interestingly, in $\mathrm{G} 2 / \mathrm{M}$ phase: compared with the control group, IR group had obvious cell cycle arrest, while IM and combination group had no cell cycle arrest.In order to highlight the independent effect of IR and $\mathrm{IM}$, the concentration of $\mathrm{IM}$ was reduced to $0.5 \mathrm{uM}$ and increased to $8 \mathrm{uM}$, and the dose of IR was increased to $150 \mathrm{~Gy}$. The cell ratio of $I M=0.5 u M+I R=150$ gy and $I M=8 u M+I R=100 G y$ in $G 0 / G 1$ and $G 2 / M$ phases was observed. The statistical results suggested that (Extended Data Fig2A, B, C). IR mainly induced cell cycle arrest (in S phase and G2 / M phase), while IM could promote apoptosis of IR induced cells in cell cycle arrest.

\section{The effect of combined action on the transcription level of related genes}

Combined with related studies and KEGG signaling pathway, radiation-related DNA damage response signaling pathway is closely related to $p 53$ signaling pathway, which mainly includes $\gamma-H 2 A X, A T M / A T R$, p53, etc. [34-35].Therefore, we speculate that IM may play a role in radiosensitization by inhibiting the above signal pathway. Therefore, at the transcription level, we studied the effect of combined action on the expression of radiation-related DNA damage repair genes (ATM, p53 and Rad51). The results showed that (Figure 6): except for the p53 combined group $(P=0.208)$ (Figure 6B), the intervention group could promote the expression of ATM,p53 and Rad51 (Figure 6A-C):;Compared with IR alone, IM combined with IR could promote ATM expression(Figure 6A), while IM combined with IR resulted in the decrease of p53 and Rad51 expression(Figure 6B,6C).

Based on the mechanism of IM as radiosensitizer in other tumors, we speculate that Rad51 may play a key role in the process of IM sensitization IR [35,36]. Therefore, we focus on the transcription of Rad51.In a certain range of IM concentration (1-4uM), with the increase of IM concentration, the inhibition of Rad51 expression was enhanced, indicating that IM could indeed inhibit Rad51 transcription, and then sensitize IR to the damage of GIST-882 cells;Interestingly, when the IM concentration exceeds the extreme value (8uM), the transcription level of Rad51 rises again. We suspect that under the action of ultra-high IM concentration, the surviving cell groups may have universal drug resistance, and maintain the stability of tumor cell genome through high expression of Rad51 (Extended Data Fig3A);However, under a certain dose of IR, the transcription level of Rad51 is generally increased, indicating that Rad51 protein is indeed 
one of the proteins that lead to radiation tolerance, which is consistent with other studies [34-36]. However, high dose of IR (200Gy) also leads to the decline of Rad51 transcription level (Extended Data Fig3B), indicating that high dose of radiation makes it difficult for cells to complete self-healing and eventually lead to apoptosis.

Compared with the single IR group, the transcriptional level of Rad51 in the combination group was inhibited (Figure6D, 6E, 6F), which indicated that IM could inhibit the increase of transcriptional level of Rad51 stimulated by IR, so as to inhibit DNA repair induced by IR and achieve sensitization.

\section{The effect of combination on the translation level of related genes}

IM, as a tyrosine kinase inhibitor, mainly suppresses PI3K / AKT and RAS-MAPK, two classic signaling pathways. We wonder whether these two tumor proliferation related signaling pathways can amplify the intensity of p53, a radiation-related DNA damage repair signaling pathway, and then maintain the stability of tumor genetic material.We found that IM did inhibit the activation of AKT / PI3K and Raf-MAPK signaling pathways (Figure 6G, H), while IR promoted the expression of ATM, y-H2AX, p53, Rad51, which are DNA double strand break repair proteins.Compared with IR alone, the combined effect promoted the expression of ATM and $y-\mathrm{H} 2 \mathrm{AX}$, but inhibited the expression of $\mathrm{p} 53$ and Rad51 protein (Figure 6G, I), which indirectly suggested that IM might inhibit its classical proliferation related signaling pathway, As a result, p53, a radiation-related DNA repair signal pathway, could not be fully activated, and its downstream DNA damage repair protein (Rad51) could not be fully activated and raised, thus enhancing the killing effect of radiation on GIST-882 cells.

\section{Discussion}

The tolerance of tumor is a great challenge for tumor therapy. The instability of tumor genome is considered to be the main reason for the resistance of tumor therapy (37). Therefore, in the process of tumorigenesis and development, DNA damage repair protein needs to be highly expressed to maintain the instability of tumor genome (38-40).In this experiment, the expression of DNA damage repair protein in GIST was significantly increased, and the high expression of Rad51 was positively correlated with the risk of GIST, which not only suggested that the high expression of DNA repair protein played an important role in the instability of GIST genome, but also led to the high risk of recurrence and drug resistance of GIST, which provided a molecular theory for IM combined with radiotherapy.Unfortunately, there are few samples of new adjuvant therapy before operation, especially the application of radiation in GIST, so it is impossible to obtain the influence of IM and IR on the expression of DNA repair protein in GIST, which is also the direction of further research in the future.

The EC50 of IR in this study is much higher than the effective radiation dose (5-40Gy) of other tumor cell lines, which fully reflects the radiosensitivity of GIST as a soft tissue sarcoma. Because the current reports on the IR study of GIST cell line are basically blank, a lot of time and energy have been spent in the early stage to explore the effective dose, and it is hoped that this study can provide a powerful dose reference for the research in this direction. In vitro cell proliferation experiment, we found that both the 
interaction of factorial analysis and synergistic index analysis, IM combined with IR showed a good synergistic inhibition effect on cells, especially IM significantly changed EC50 in IR group, which may greatly enhance the response rate of clinically insensitive patients under the action of IM sensitizer, and may break the guide of GIST radiosensitivity Concept has great clinical significance.In addition, in cell cycle and apoptosis experiments, IM can rapidly induce apoptosis without obvious cell cycle arrest effect, reflecting the sensitivity of target drugs. IR mainly leads to cell cycle arrest. At this time, cells stimulate DNA damage repair protein expression through feedback, repair DNA damage caused by IR, and lead to radiation tolerance. The results of immunohistochemistry showed that these proteins were highly expressed in GIST, which further explained the molecular basis of radiosensitivity in GIST.

At the molecular level, previous studies of KEGG cell pathway analysis and a large number of related IM combined with IR in other tumors have been reported, We speculate that when the cell is damaged by IR, it activates the DNA radiation damage response axis in the upstream of p53 signal pathway with ATM /ATR- $y-H 2 A X-p 53$ as the axis, and then collects the downstream related proteins with Rad51 protein as the core to focus on the vicinity of the damaged DNA double strand, and repair the broken DNA double strand through the HR signal pathway,this downstream repair response is called the radiation damage repair axis(Extended Data Fig4A,B).By analyzing the cumulative enhancement effect of IM combined with IR on the transcription level of upstream ATM, and IM can inhibit the transcription of p53 and Rad51 in IR group, it shows that the joint damage to cells stimulates the stress response of ATM, the upstream protein, while IM inhibits the activation of DNA repair protein caused by IR, which occurs in the signal molecules below p53. Therefore, we boldly suspect the signal pathway and IR induced by IM The repair signal pathway of hair may intersect on p53 protein, and the downstream Rad51 protein may be the key star molecule of IM combined with IR to inhibit radiation repair.Single dose cumulative effect analysis of Rad51 transcription level showed that IM promoted Rad51 transcription compared with the control group, which was contrary to our previous expectation, and Rad51 transcription level did gradually decrease with the increase of IM concentration, so we think that under the range of IM treatment concentration, cell damage effect was mainly shown, the signal pathway to maintain the stability of tumor cell genome occupied the dominant position resulted in the up regulation of Rad51 transcription level.However, with the increase of IM concentration, the signal of IM inhibiting radiation damage gradually increased and occupied the dominant position, leading to the downregulation of Rad51 transcription.However, at a certain dose of IR, the transcription level of Rad51 was generally increased, which was consistent with our expectation. In the combination group of different doses, IM can inhibit the expression of Rad51, which indirectly shows that IM can inhibit the expression of DNA repair protein induced by IR by inhibiting the expression of Rad51 protein.In GIST, IM mainly suppresses PI3K/AKT and Raf-MAPK. Therefore, we speculate whether the inhibition of these two tumor proliferation signaling pathways leads to the attenuation of DNA damage repair signaling pathway. Protein analysis shows that IM combined with IR indeed leads to the accumulation of upstream ATM and $\mathrm{Y}-\mathrm{H} 2 \mathrm{AX}$ damage response protein, while IM can inhibit IR induced p53 and Rad51 homologous recombination DNA Expression of a repair protein.

In the future, there are still many questions to be discussed about the synergistic inhibition mechanism of IM combined with IR on GIST, such as whether the synergistic effect of in vivo animal model is as 
significant as that of in vitro cell experiment? Whether Rad51 is the key star molecule of IM combined with $I R$, it is far from enough to draw a conclusion only from the apparent expression.

\section{Conclusions}

IM combined with IR can synergistically inhibit the proliferation, apoptosis, clonogenesis, invasion, migration and cell cycle of GIST cells. The mechanism of synergism may be through IM to inhibit p53, a signal pathway of radiation damage repair.

\section{Abbreviations}

ATM:Ataxia telangiectasia-mutated gene;ATR:Ataxia-telangiectasia mutated and RAD3-related; BSA:bovine serum albumin; Cl:combination index; Gy:Gray; GIST Gastrointestinal Stromal Tumor; IM:Imatinib;IR:Iradiation;RT:radiotherapy; uM:umol/l;

\section{Declarations}

Ethics approval and consent to participate:Not applicable;

Consent for publication:Yes;

Availability of data and materials:All data generated or analysed during this study are included in this published article and its supplementary information files;

Competing interests:There are no conflicts of interest or financial ties to disclose from any of author; Funding:Climbing Plan of Fujian Provincial Department of Science and Technology(2017Y9040)

Authors' contributions:Wenbiao Xiao and Yongjian Zhou came up with the study concept and Wenbiao Xiao developed the study design and protocol. Wenbiao Xiao completed most of the experimental tasks.Guosheng Lin collected the study data. All authors read and approved the final manuscript.

Acknowledgements:This study was supported by Laboratory of Transformation, Fujian Medical University And Institute of Life Sciences, Fuzhou University.

\section{Authors' information (optional):}

Wenbiao Xiao:Intensive Care Unit,The First Affiliated Hospital of Fujian Medical University, No.20 Chating Zhong Road,Tai jiang District, Fuzhou 350001, Fujian Province, China;Telephone:15060043011;

Yongjian Zhou:Department of Gastric Surgery, Fujian Medical University Union Hospital, No.29 Xin quan Road, Fuzhou 350001, Fujian Province, China;Telephone:18850713045;zhouyj2019@163.com; 
Guosheng Lin:Department of Gastric Surgery, Fujian Medical University Union Hospital, No.29 Xin quan Road, Fuzhou 350001, Fujian Province, China;Telephone: +86-591-83363366;

Renjie Guo:Department of Gastric Surgery, Fujian Medical University Union Hospital, No.29 Xin quan Road, Fuzhou 350001, Fujian Province, China;Telephone: +86-591-83363366; 523840366@qq.com;

\section{References}

[1] Heinrich M C, Corless C L, Duensing A, et al. PDGFRA activating mutations in gastrointestinal stromal tumors[J]. Science, 2003, 299(5607): 708-710.

[2] Nakul Valsangkar, MD, Amikar Sehdev, et al. Current management of gastrointestinal stromal tumors:Surgery, current biomarkers, mutations, and therapy [J]. Surgery ,2015囚158:1149-64.

[3]Antonescu C R. Adjuvant imatinib mesylate after resection of localised, primary gastrointestinal stromal tumour: a randomised, double-blind, placebo-controlled trial.[J]. Lancet, 2009, 373(9669):10971104.

[4] Blanke CD, Demetri GD, von Mehren M, et al. Long-term results from a randomized phase II trial of standard- versus higher-dose imatinib mesylate for patients with unresectable or metastaticgastrointestinal stromal tumors expressing KIT. J Clin Oncol. 2008;26:620-625.

[5] ESMO/European Sarcoma Network Working Group. Gastrointestinal stromal tumors: ESMO Clinical Practice Guidelines for diagnosis, treatment and follow-up. Ann Oncol 2014;25(Suppl 3):iii21-6

[6] Zhang Q, Xu J, Qian Y, et al. Association of imatinib plasma concentration and single nucleotide polymorphisms with adverse drug reactions in patients with gastrointestinal stromal tumors. Mol Cancer Ther. 2018;17:2780-2787

[7] Bauer S, Joensuu H, et al. Emerging Agents for the Treatment of Advanced, Imatinib-Resistant Gastrointestinal Stromal Tumors: Current Status and Future Directions. Drugs 2015;75(12):1323-34.

[8] Takahashi T, Elzawahry A, Mimaki S, et al. Genomic and transcriptomic analysis of imatinib resistance in gastrointestinal stromal tumors. Genes Chromosomes Cancer. 2017;56:303-313.

[9] 2017NCCN Clinical Practice Guidelines(V2).

[10]Crosby JA, Catton CN, et al.Malignant gastrointestinal stromal tumors of the small intestine:a review of 50 cases from a prospective database. Ann Surg Oncol.2001;8(1):50-9.

[11]Pierie JP, Choudry U, et al. The effect of and surgery grade on outcome of gastrointestinal stromal tumors.Arch Surg.2001; 136(4):383-9. 
[12]Aparicio S, Caldas C. The implications of clonal genome evolution for cancer medicine. N Engl J Med. 2013;368(9):842-851.

[13]Di Scioscio V, Greco L, Pallotti MC, et al. Three cases of bone metastases in patients with gastrointestinal stromal tumors. Rare Tumors.2011;3(2):e17.

[14]Hurwitz J, Constantinidou A, Saran F, et al. The role of radiotherapy in metastatic gastrointestinal stromal tumor (GIST). Proc Connect Tissue Oncol Soc. 2008(2008): abst 35023.

[15]Benjamin D. Goodman DO , Edward M. Mannina MD, et al. Long-term safety and efficacy of stereotactic body radiation therapy for hepatic oligometastases. Practical Radiation Oncology (2015) xx, $x x x-x x x$.

[16]Corbin KS, Hellman S, Weichselbaum RR. Extracranial oligometastases:a subset of metastases curable with stereotactic radiotherapy.J Clin Oncol. 2013;31(11):1384-1390.

[17] Gatto et al. Radiotherapy in the management of gist: state of the art and new potential scenarios.Clin Sarcoma Res (2017) 7:1.

[18] Takeshi Gohongi, Hiroyuki lida, et al. Postsurgical radiation therapy for gastric carcinosarcoma with c-kit expression: A case report. World J Gastroenterol 2015 ;21(9): 2830-2835.

[19] Joshua Halpern,Yong-June Kim,et al.Effectiveness of radiation therapy in GIST:A case report.Journal of Gastrointestinal Oncology. 2012;2(3):143-146.

[20] Cem Borubana, Orhan Sencanb, et al. Metastatic gastrointestinal stromal tumor with long-term response after treatment with concomitant radiotherapy and imatinib mesylate.Anti-Cancer Drugs. 2007;18:969-972.

[21] Kimberly S Corbin1, et al. Considering the role of radiation therapy for gastrointestinal stromal tumor OncoTargets and Therapy 2014:7 713-718.

[22] Cuaron JJ, Goodman KA, Lee N, Wu AJ. External beam radiation therapy for locally advanced and metastatic gastrointestinal stromal tumors. Radiat Oncol. 2013;8(1):274.

[23] Joensuu H, Eriksson M, Collan J, Balk MH, Leyvraz S, Montemurro M.Radiotherapy for GIST progressing during or after tyrosine kinase inhibitor therapy: a prospective study. Radiother Oncol. 2015;116(2):233-8.

[24]Radujkovic, A.Dreger, P.Hegenbart, et al. Imatinib-supplemented myeloablative total-body irradiation/cyclophosphamide conditioning prior to allogeneic stem cell transplantation as consolidation treatment in patients with blast crisis of chronic myeloid leukemia. Eur J Haematol. 2014;6(92): 546-9 
[25]Kaygusuz-Atagunduz, I.Toptas, T,et al. Newly diagnosed breast cancer in a patient receiving imatinib mesylate. J Cancer Res Therc. 2014;4(10): 1107-8

[26]Qiao, B.Kerr, M.Groselj, B,et al. Imatinib radiosensitizes bladder cancer by targeting homologous recombination. Cancer Res. 2013;5(73): 1611-20.

[27]De Moura, L. R.Marshall, J. C,et al. The effect of imatinib mesylate on the proliferation, invasive ability, and radiosensitivity of retinoblastoma cell lines. 2013;(27): 92-9.

[28]Podtcheko A, Ohtsuru A, et al. Inhibition of ABL tyrosine kinase potentiates radiation-inducedterminal growth arrest in anaplastic thyroid cancer cells. Radiat Res. 2006;165(1):35-42.

[29]Choudhury A, Zhao $\mathrm{H}$, et al. Targeting homologous recombination using imatinib results in enhanced tumor cell chemosensitivity and radiosensitivity. Mol Cancer Ther.2009;8(1):203-13.

[30]Zhu, J.Zhou, L.Wu, G, et al. A novel small molecule RAD51 inactivator overcomes imatinib-resistance in chronic myeloid leukaemia. EMBO Mol Med. 2013;3(5): 353-65.

[31]Mukhopadhyay Asima,Drew Yvette,Matheson Elizabeth et al. Evaluating the potential of kinase inhibitors to suppress DNA repair and sensitise ovarian cancer cells to PARP inhibitors.[J] .Biochem. Pharmacol., 2018, undefined: undefined.

[32]Ferguson Peter J,Vincent Mark D,Koropatnick James,Synergistic Antiproliferative Activity of the RAD51 Inhibitor IBR2 with Inhibitors of Receptor Tyrosine Kinases and Microtubule Protein.[J] .J. Pharmacol. Exp. Ther., 2018, 364: 46-54.

[33]Zhu Jiewen,Zhou Longen,Wu Guikai et al. A novel small molecule RAD51 inactivator overcomes imatinib-resistance in chronic myeloid leukaemia.[J] .EMBO Mol Med, 2013, 5: 353-65.

[34]Tarrade Stephane,Bhardwaj Tanya,Flegal Matthew et al. Histone H2AX Is Involved in FoxO3aMediated Transcriptional Responses to lonizing Radiation to Maintain Genome Stability.[J] .Int J Mol Sci, 2015, 16: 29996-30014.

[35]King Harry O,Brend Tim,Payne Helen L et al. RAD51 Is a Selective DNA Repair Target to Radiosensitize Glioma Stem Cells.[J] .Stem Cell Reports, 2017, 8: 125-139.

[36]Ferguson Peter J,Vincent Mark D,Koropatnick James,Synergistic Antiproliferative Activity of the RAD51 Inhibitor IBR2 with Inhibitors of Receptor Tyrosine Kinases and Microtubule Protein.[J] .J. Pharmacol. Exp. Ther., 2018, 364: 46-54.

[37]Lawrence, M. S. et al. Mutational heterogeneity in cancer and the search for new cancer-associated genes. Nature 499, 214-218 (2013). 
[38]Tang Siyuan,Liu Bailong,Liu Min et al. Ionizing radiation-induced growth in soft agar is associated with miR-21 upregulation in wild-type and DNA double strand break repair deficient cells.[J] .DNA Repair (Amst.), 2019, 78: 37-44.

[39]Biermann Jana,Langen Britta,Nemes Szilárd et al. Radiation-induced genomic instability in breast carcinomas of the Swedish haemangioma cohort.[J] .Genes Chromosomes Cancer, 2019, undefined: undefined.

[40]Otsubo Ryota,Matsuda Katsuya,Mussazhanova Zhanna et al. A novel diagnostic method for thyroid follicular tumors based on immunofluorescence analysis of p53-binding protein 1 expression: detection of genomic instability.[J] .Thyroid, 2019, undefined: undefined.

\section{Tables}

Table 1 Correlation between Rad51 protein and clinicopathological data of GIST patients 


\section{Related factors Rad51 positive expression rate \\ $\square^{2} \quad P$ value}

Gender

1.621

0.264

male $\quad 47 / 54(87.40 \%)$

female 25/26 (96.15\%)

Age (years)

$1.455 \quad 0.275$

$$
\begin{aligned}
& <55 \\
& \geq 55 \quad 43 / 46(90.38 \%)
\end{aligned}
$$

Longest diameter (CM)

$1.270 \quad 0.455$

$$
\begin{array}{lll}
\leq 5 & & 30 / 35(85.71 \%)
\end{array}
$$

Mitotic image (Per / 50HP)

$2.769 \quad 0.188$

$$
\begin{array}{ll}
\leq 5 & 53 / 61(86.89 \%) \\
>5 & 19 / 19(100.00 \%)
\end{array}
$$

CD117

$9.202 \quad 0.019$

positive

$68 / 73(93.15 \%)$

negative $4 / 7(57.14 \%)$

CD34

$0.044 \quad 1.00$

positive

negative

DOG-1
$61 / 68$ ( $89.71 \%)$

$11 / 12(91.67 \%)$

$1.003 \quad 0.434$ 
positive

negative

Risk degree

Low risk

Middle risk

High risk

Tumor site

stomach

non stomach
$51 / 58$ ( $87.93 \%$ )

$21 / 22$ ( $95.45 \%$ )

4.205

0.048

$29 / 35$ ( $82.86 \%$ )

$8 / 9$ ( $88.89 \%)$

$35 / 36$ ( $97.22 \%$ )

$0.000 \quad 1.000$

$36 / 40$ ( $90.00 \%)$

$36 / 40(90.00 \%)$

Table 2 Chou-talalayAnalysis of Cl value of combined inhibition of IM and IR on GIST-882 cells

\begin{tabular}{|clll|}
\hline Concentration & \multicolumn{3}{l|}{$\mathrm{IM}(\mathrm{uMol} / \mathrm{L})$} \\
\hline $\mathrm{IR}(\mathrm{Gy})$ & 1 & 5 & 10 \\
\hline 30 & 0.42203 & 0.49320 & 0.38266 \\
\hline 80 & 0.26909 & 0.25183 & 0.26664 \\
\hline 180 & 0.30412 & 0.22518 & 0.11184 \\
\hline 240 & 0.29569 & 0.12060 & 0.09823 \\
\hline
\end{tabular}

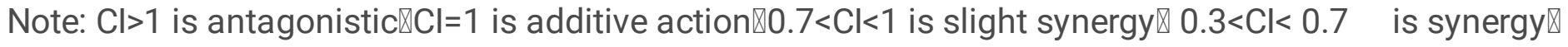
$\mathrm{Cl}<0.3$ is strong synergy.

Figures 

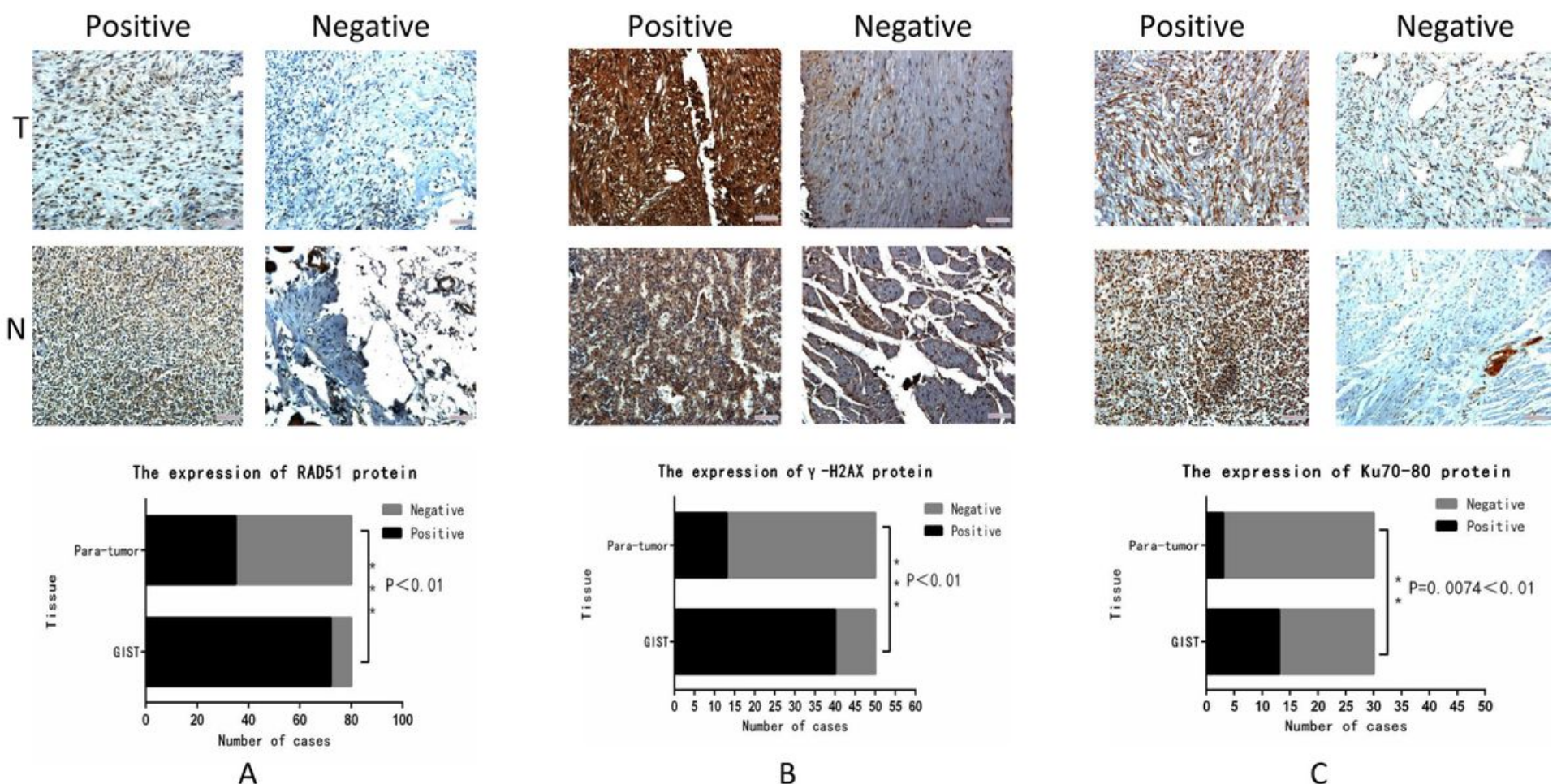

A

B

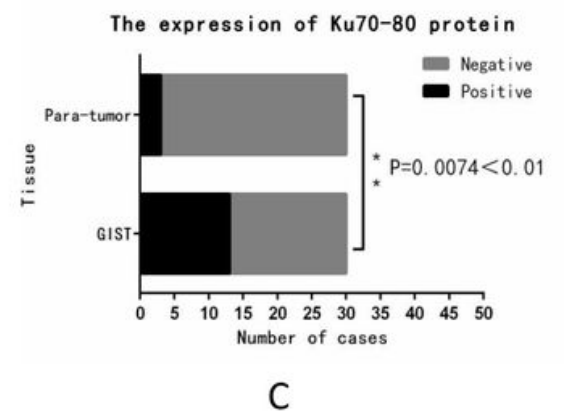

Figure 1

\section{Figure 1}

$A, B$ and $C$ respectively show that: the high expression (left) and low expression (right) of Rad51 protein, $\mathrm{Y}$-H2A.X protein and Ku70/80 protein in gastrointestinal stromal tumor (upper) and its adjacent tissues (lower), the difference is statistically significant (p1<0.01, p2<0.01, p3<0.01); microscope multiple: 400x;

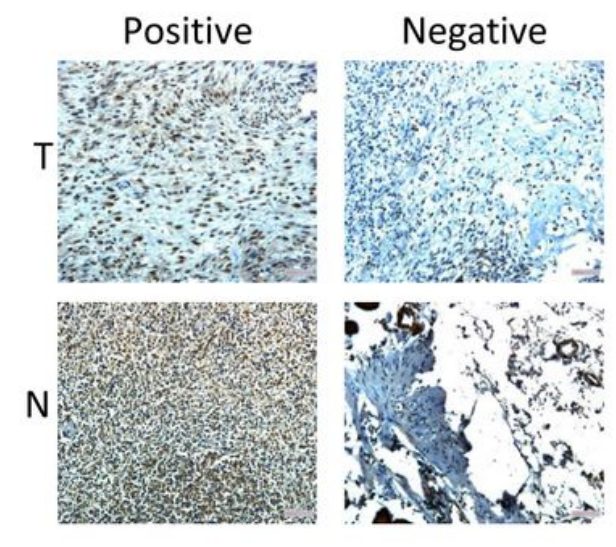

The expression of RAD51 protein

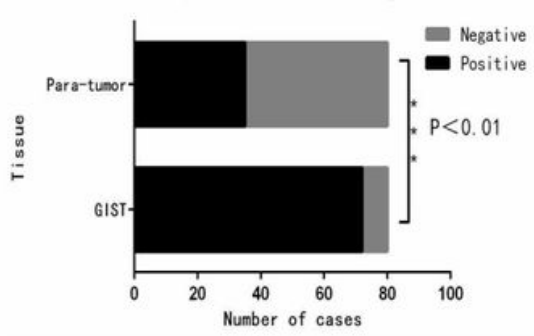

A
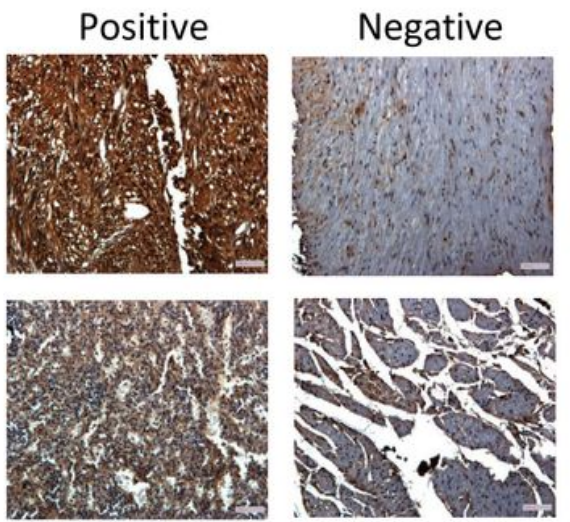

The expression of $y-H 2 A X$ protein

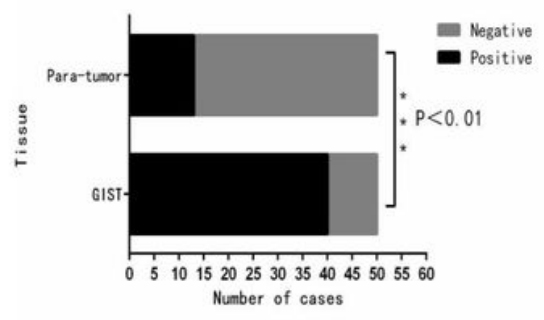

B
Positive
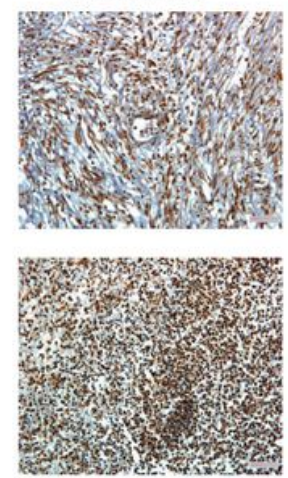

Negative
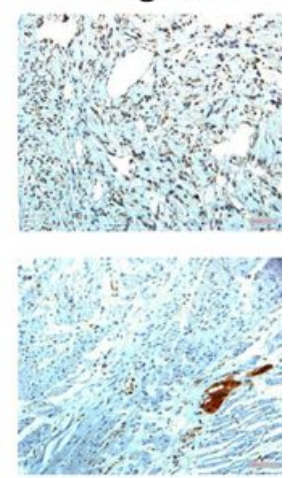

The expression of Ku70-80 protein

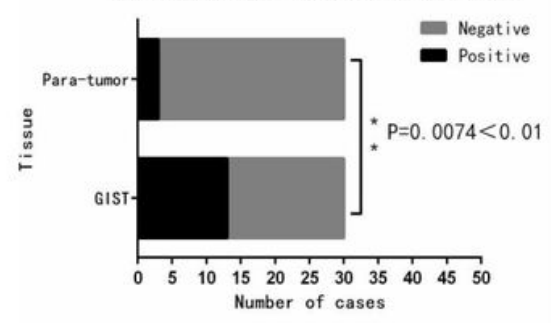

C

Figure 1 


\section{Figure 1}

$A, B$ and $C$ respectively show that: the high expression (left) and low expression (right) of Rad51 protein, $\mathrm{Y}$-H2A.X protein and Ku70/80 protein in gastrointestinal stromal tumor (upper) and its adjacent tissues (lower), the difference is statistically significant (p1<0.01, p2<0.01, p3<0.01); microscope multiple: 400x;

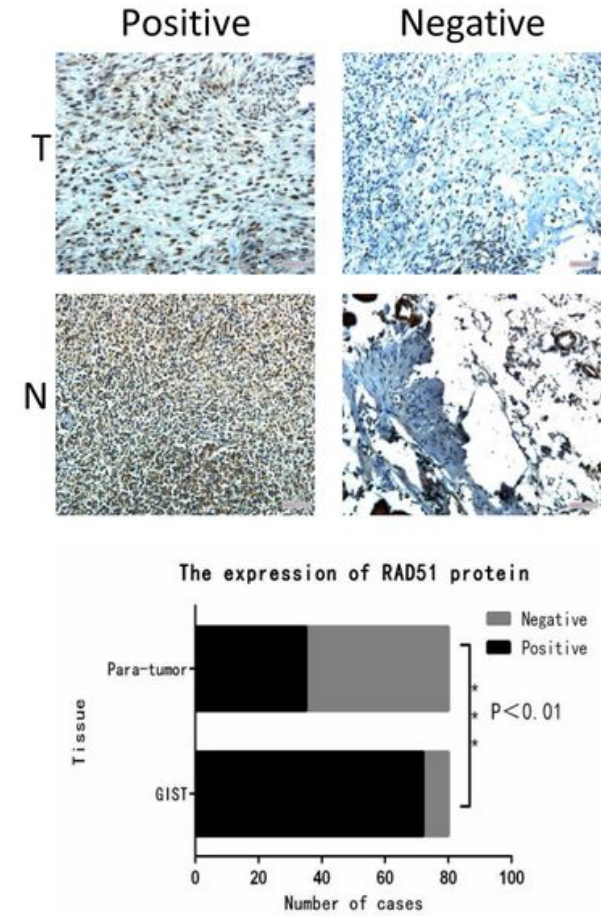

A
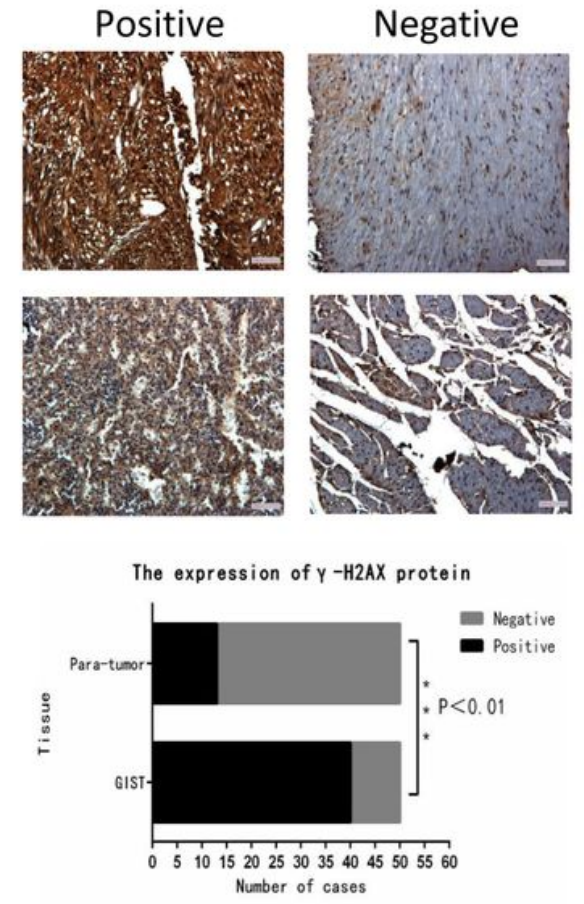

B
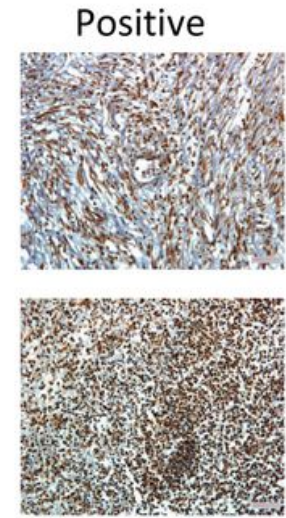

Negative
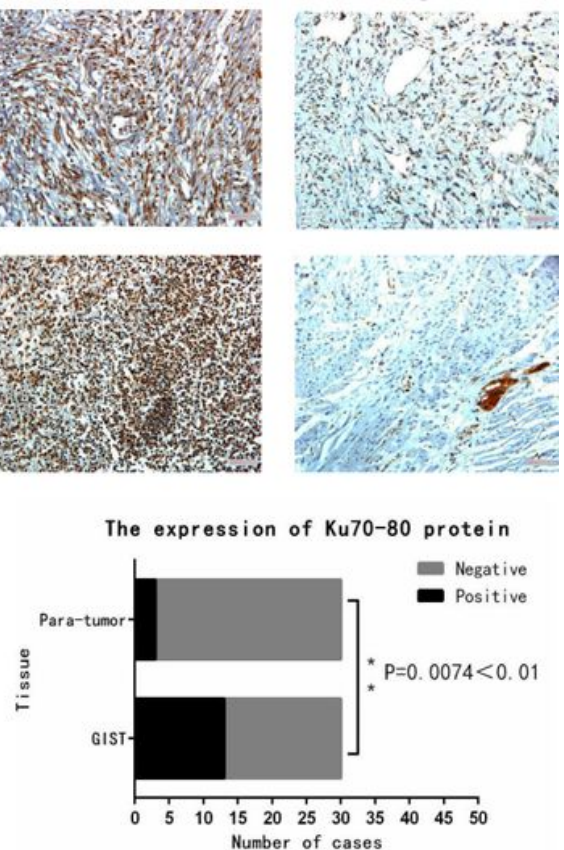

C

Figure 1

\section{Figure 1}

$A, B$ and $C$ respectively show that: the high expression (left) and low expression (right) of Rad51 protein, $\mathrm{Y}$-H2A.X protein and Ku70/80 protein in gastrointestinal stromal tumor (upper) and its adjacent tissues (lower), the difference is statistically significant (p1<0.01, p2<0.01, p3<0.01); microscope multiple: 400x; 


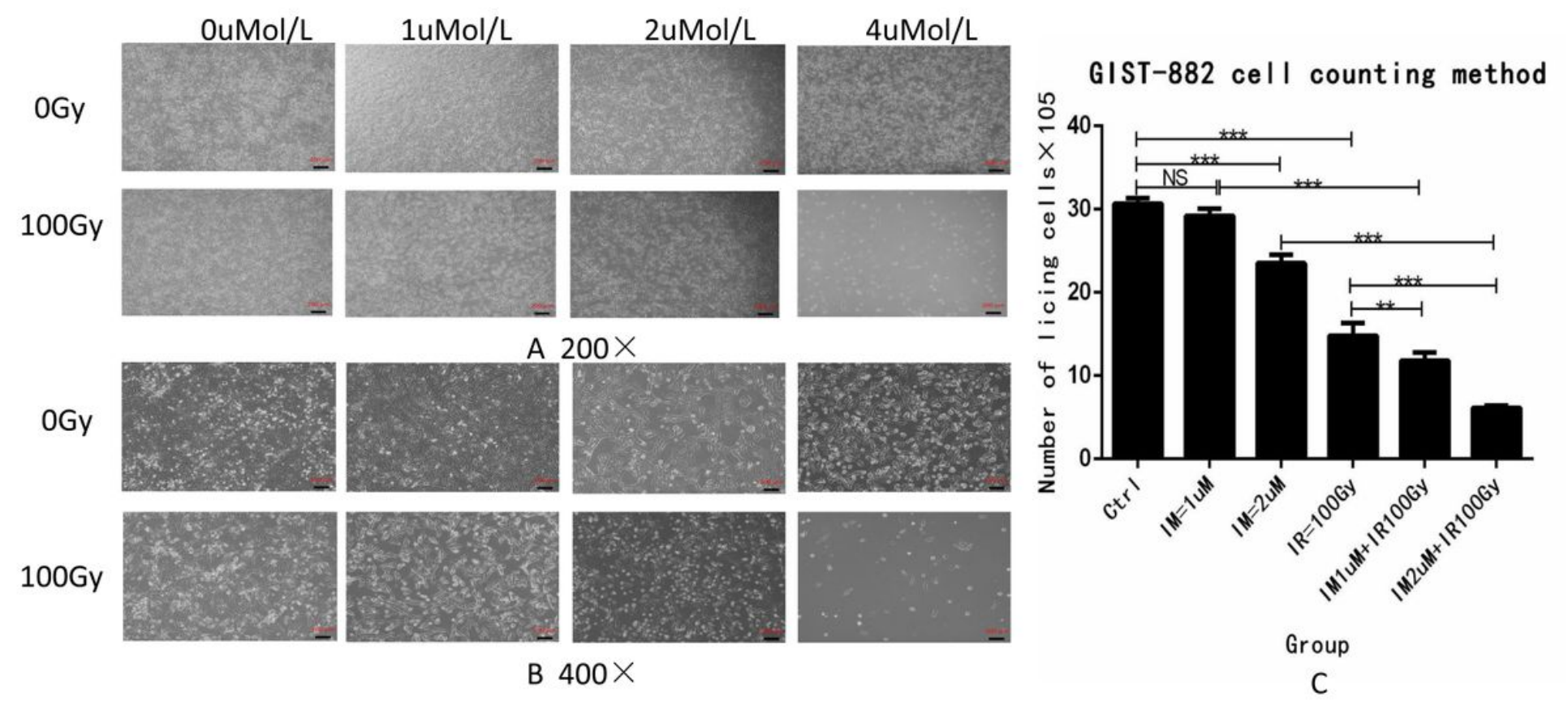

Figure 2

\section{Figure 2}

$A \square B$ respectively show that the effects of different combinations of $I R=100 \mathrm{~Gy}$ and $I M=0,1,2$ and $4 \mathrm{uM}$ on the morphology of GIST-882 cells were observed under 200 and 400 power microscope; C:the effects of IR $=100 \mathrm{~Gy}, \mathrm{IM}=1$ and $2 \mathrm{uM}$ on the number of GIST-882 cells were detected by the method of living cell count; Comparison among groups:F=317.35, Sig=0.000区 LSD Multiple comparisons: Ctrl VS IM=1: Sig=0.088》 Ctrl VS IR=100: Sig=0.000冈 IM=1 VS United 1: Sig=0.000冈 IR=100Gy VS United 1: Sig=0.003囚 Ctrl VS

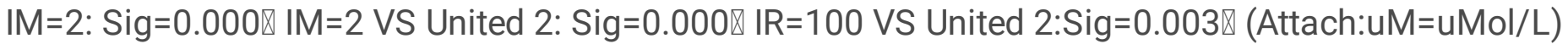




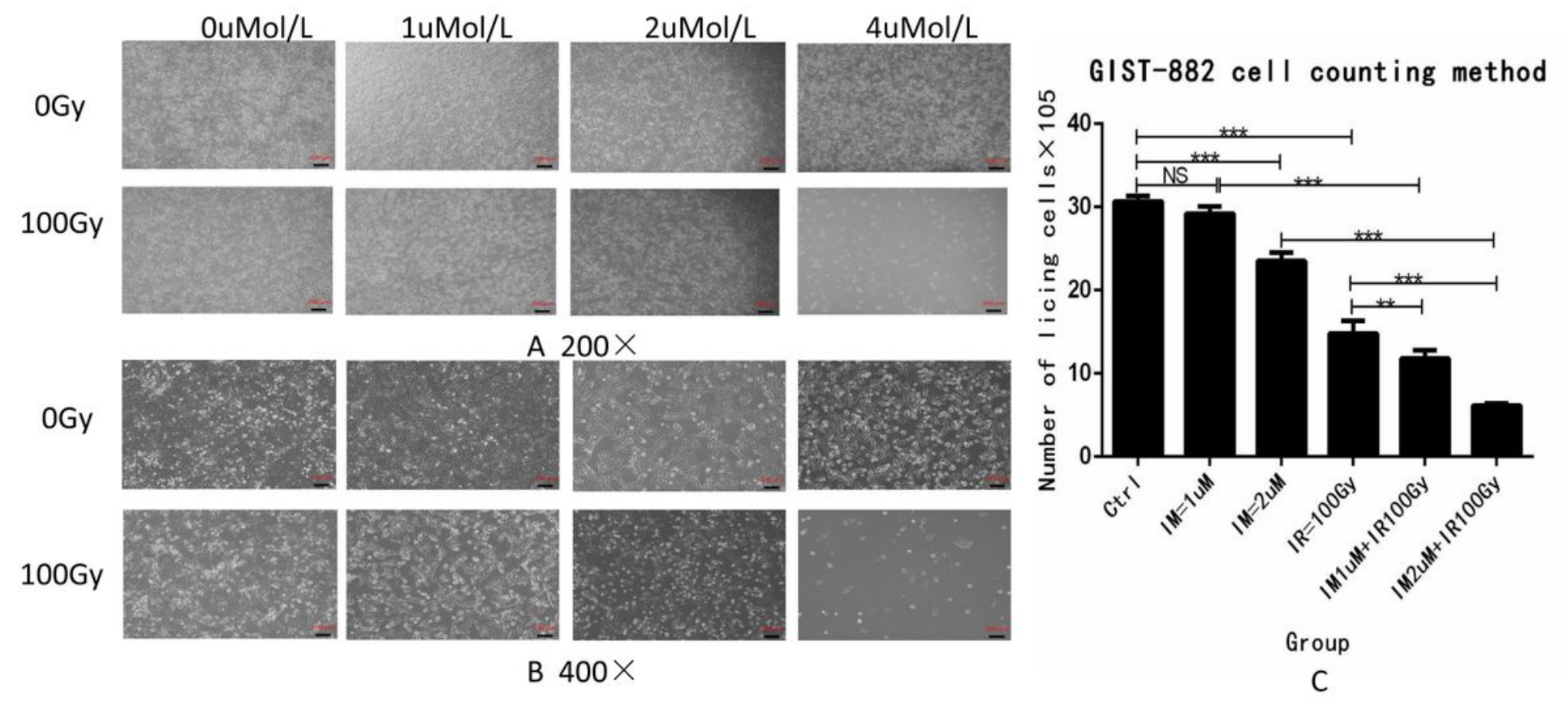

Figure 2

\section{Figure 2}

$A \square B$ respectively show that the effects of different combinations of $I R=100 \mathrm{~Gy}$ and $I M=0,1,2$ and $4 \mathrm{uM}$ on the morphology of GIST-882 cells were observed under 200 and 400 power microscope; C:the effects of IR $=100 \mathrm{~Gy}, \mathrm{IM}=1$ and $2 \mathrm{uM}$ on the number of GIST-882 cells were detected by the method of living cell count; Comparison among groups:F=317.35, Sig=0.000区 LSD Multiple comparisons: Ctrl VS IM=1: Sig=0.088》 Ctrl VS IR=100: Sig=0.000冈 IM=1 VS United 1: Sig=0.000冈 IR=100Gy VS United 1: Sig=0.003囚 Ctrl VS

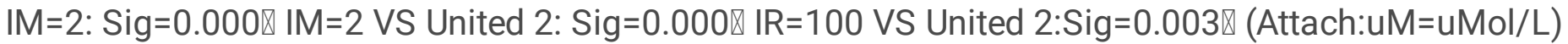




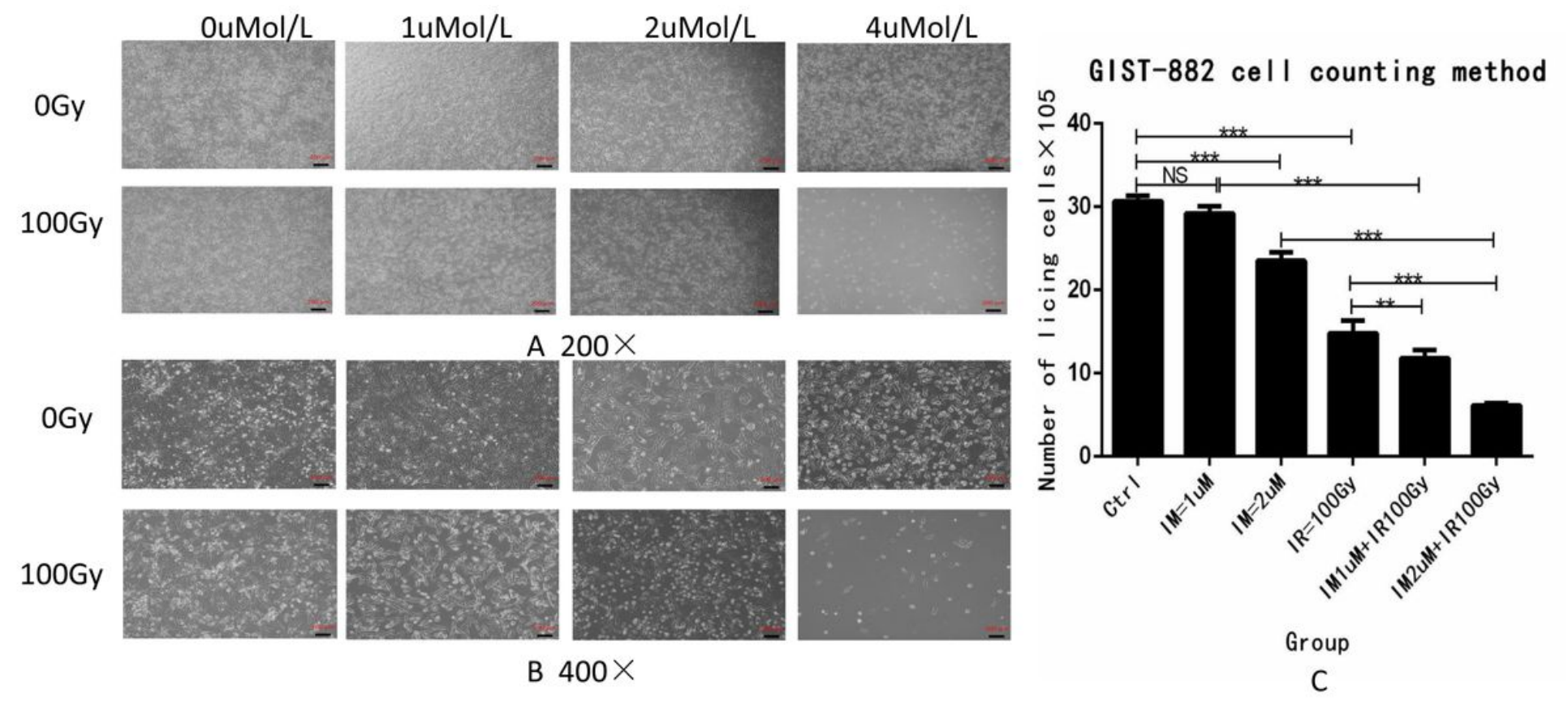

Figure 2

\section{Figure 2}

$A \square B$ respectively show that the effects of different combinations of $I R=100 \mathrm{~Gy}$ and $I M=0,1,2$ and $4 \mathrm{uM}$ on the morphology of GIST-882 cells were observed under 200 and 400 power microscope; C:the effects of IR $=100 \mathrm{~Gy}, \mathrm{IM}=1$ and $2 \mathrm{uM}$ on the number of GIST-882 cells were detected by the method of living cell count; Comparison among groups:F=317.35, Sig=0.000 LSD Multiple comparisons: Ctrl VS IM=1: Sig=0.088 Ctrl VS IR=100: Sig=0.000冈 IM=1 VS United 1: Sig=0.000冈 IR=100Gy VS United 1: Sig=0.003囚 Ctrl VS $I M=2:$ Sig=0.000冈 IM=2 VS United 2: Sig=0.000冈 IR=100 VS United 2:Sig=0.003\ (Attach:uM=uMol/L)
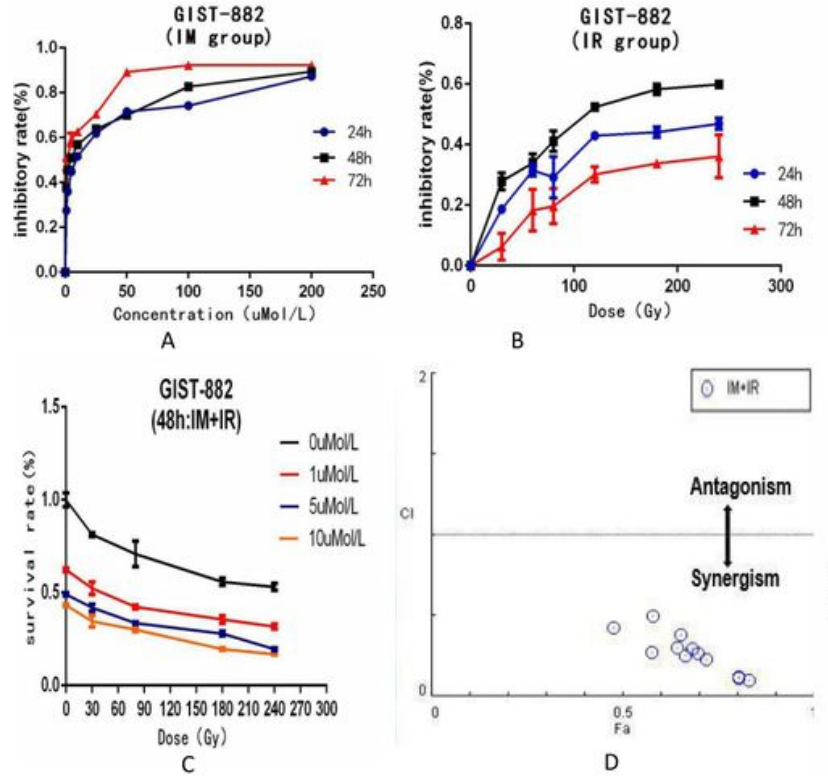

GIST-882 cell clone formation asssy

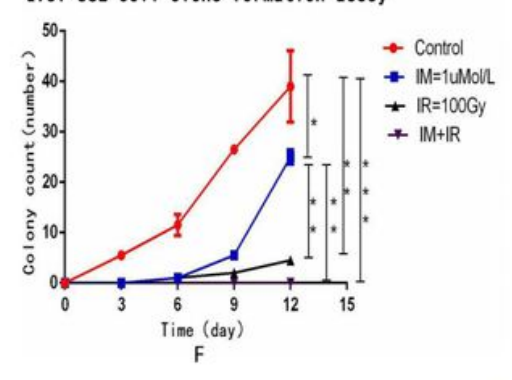

The effect of IM on IR's EC50

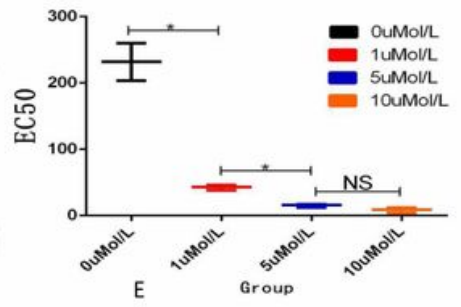

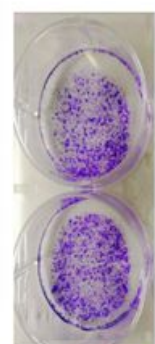
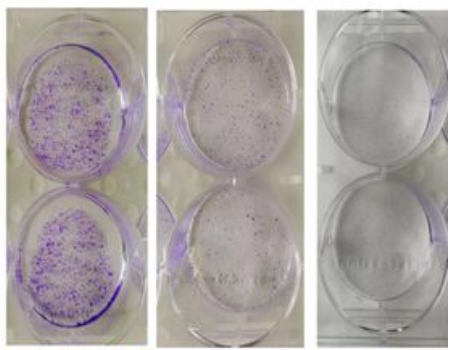

敨

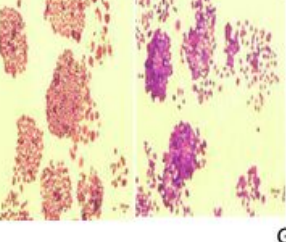

Figure 3 


\section{Figure 3}

CCK-8 was used to study the inhibitory effect of IM and IR on the proliferation of GIST-882 cells,

FigureA:The time and Concentration dependence curve of IM on GIST- 882 cells, the "Mauchly" spherical symmetry test:W=0.945 P=0.525;Concentration $F=3824.56 \mathrm{P}=0.000$; Time $\mathrm{F}=1469.668 \mathrm{P}=0.000 \otimes$ The interaction between IM concentration and time: Im * time F=25.596 P=0.000冈 FigureB. "Mauchly" spherical symmetry test:W=0.907 $\mathrm{P}=0.483$;Concentration $\mathrm{F}=118.96 \mathrm{P}=0.000$;Time: $\mathrm{F}=201.41$ $\mathrm{P}=0.000$; Interaction of $I R$ dose and time $\mathbb{I} \mathrm{R}^{\star} \mathrm{Time} \mathrm{F}=1.41 \mathrm{P}=0.215 \otimes$ FigureC, Correction model of ANOVA in factorial design $F=150.07 \mathrm{Sig}=0.000 \otimes \mathrm{IM}$ main effect $\mathrm{F}=325.54 \mathrm{Sig}=0.000 \otimes \mathrm{IR}$ main effect $\mathrm{F}=325.26$

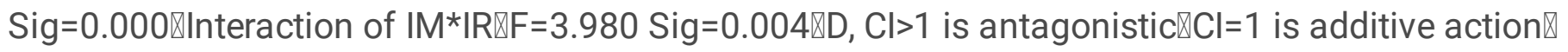
$0.7<\mathrm{Cl}<1$ is slight synergy $\mathbb{\otimes} 0.3<\mathrm{Cl}<0.7$ is synergy, $\mathrm{Cl}<0.3$ is strong synergy. Figure3E:Effect of IM on EC50 in IR group,When IM is OuM, 1uM, 5uM and 10uM,the EC50 of IR group are 231.78Gy, 37.85Gy, 15.95Gy and 8.88Gy respectively,homogeneity test of variance: $F=2.939, S i g=0.099 \llbracket$ Comparison of EC50:F=162.84(P区 0.01) $₫ T a m h a n e$ correction multiple comparison:IM=0 VS IM=1: $F=16.42(P \bowtie 0.01), I M=1$ VS IM=5: $F=2.89(P$ $\nabla 0.01), I M=5$ VS IM=10:F=2.53( $p=0.29)$ $\mathbb{F}$ igure3F,G: The effects of $I M$ alone, IR group and combination group on the clonogenesis of GIST-882 cells:One way ANOVA,LED multiple comparison $\mathbb{C}$ tr VS $I M=1 \mathrm{uM}(P=0.018), \mathrm{Ctr}$ VS IR=100Gy $(P=0.001)$, Ctr VS Combined group $(P=0.000) \otimes I M=1$ uM VS Combined group $(P=0.002), I R=100 G y$ VS Combined group $(P=0.28) \rrbracket$
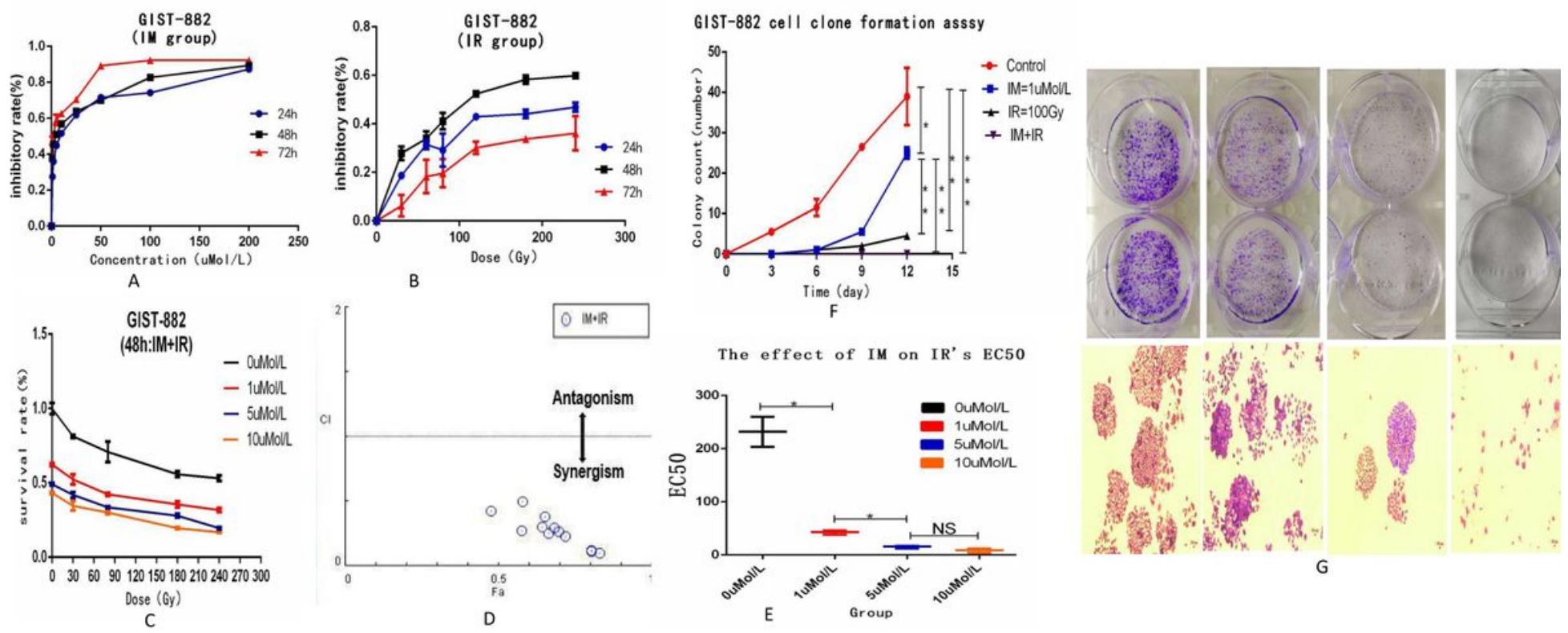

Figure 3

\section{Figure 3}

CCK-8 was used to study the inhibitory effect of IM and IR on the proliferation of GIST-882 cells, FigureA:The time and Concentration dependence curve of IM on GIST- 882 cells, the "Mauchly" spherical symmetry test:W=0.945 P=0.525;Concentration $F=3824.56 \mathrm{P}=0.000$; Time $\mathrm{F}=1469.668 \mathrm{P}=0.000 \otimes$ The interaction between IM concentration and time: Im * time $F=25.596 \mathrm{P}=0.000 \otimes$ FigureB. "Mauchly" spherical symmetry test:W=0.907 P=0.483;Concentration $F=118.96 P=0.000 ;$ Time: $F=201.41$ 
$\mathrm{P}=0.000$; Interaction of IR dose and time $\mathbb{I} \mathrm{R} *$ Time $\mathrm{F}=1.41 \mathrm{P}=0.215 \rrbracket$ FigureC, Correction model of ANOVA in factorial design $F=150.07 \mathrm{Sig}=0.000 \mathrm{VIM}$ main effect $\mathrm{F}=325.54 \mathrm{Sig}=0.000 \mathrm{QIR}$ main effect $\mathrm{F}=325.26$

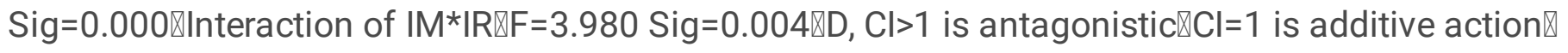
$0.7<\mathrm{Cl}<1$ is slight synergy $\mathbb{\otimes} 0.3<\mathrm{Cl}<0.7$ is synergy, $\mathrm{Cl}<0.3$ is strong synergy. Figure3E:Effect of IM on EC50 in IR group,When IM is OuM, 1uM, 5uM and 10uM,the EC50 of IR group are 231.78Gy, 37.85Gy, 15.95Gy and 8.88Gy respectively,homogeneity test of variance: $F=2.939, S i g=0.099 \otimes C o m p a r i s o n ~ o f ~ E C 50: F=162.84(P 区$ $0.01) \otimes T a m h a n e$ correction multiple comparison:IM=0 VS IM=1: $F=16.42(P \otimes 0.01), I M=1$ VS IM=5: $F=2.89(P$ $\otimes 0.01), I M=5$ VS $I M=10: F=2.53(p=0.29) \otimes F i g u r e 3 F, G$ : The effects of $I M$ alone, IR group and combination group on the clonogenesis of GIST-882 cells:One way ANOVA,LED multiple comparison $₫ \mathrm{Ctr}$ VS $I M=1 \mathrm{uM}(P=0.018)$, Ctr VS IR=100Gy $(P=0.001)$, Ctr VS Combined group $(P=0.000) \otimes I M=1$ uM VS Combined group $(P=0.002), I R=100 G y$ VS Combined group $(P=0.28) \rrbracket$
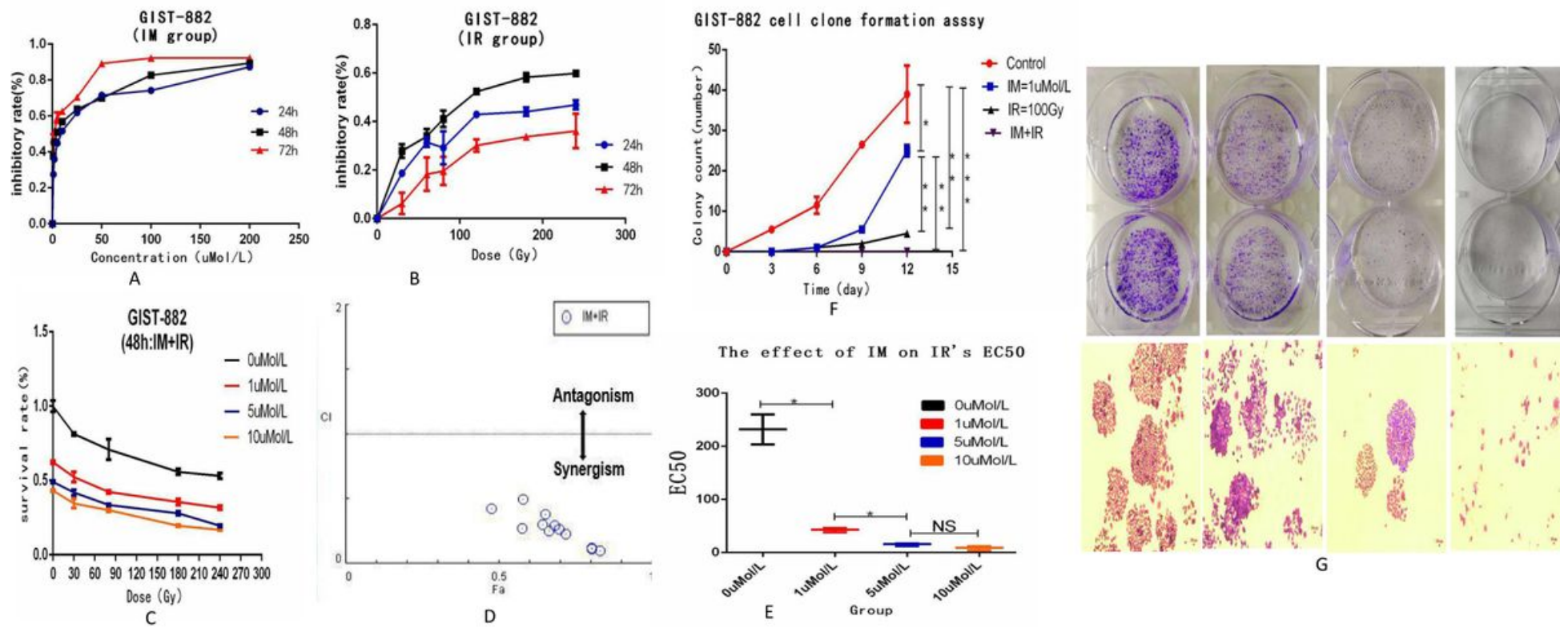

Figure 3

\section{Figure 3}

CCK-8 was used to study the inhibitory effect of IM and IR on the proliferation of GIST-882 cells, FigureA:The time and Concentration dependence curve of IM on GIST- 882 cells, the "Mauchly" spherical symmetry test:W=0.945 P=0.525;Concentration $F=3824.56 \mathrm{P}=0.000$;Time $F=1469.668 \mathrm{P}=0.000$ खThe interaction between IM concentration and time: Im * time F=25.596 P=0.000冈 FigureB. "Mauchly" spherical symmetry test:W=0.907 $P=0.483$;Concentration $F=118.96 P=0.000$;Time: $F=201.41$ $\mathrm{P}=0.000$; Interaction of $\mathrm{IR}$ dose and time $\mathbb{I} \mathrm{R}^{\star} \mathrm{T}$ Time $\mathrm{F}=1.41 \mathrm{P}=0.215 \rrbracket$ FigureC, Correction model of ANOVA in

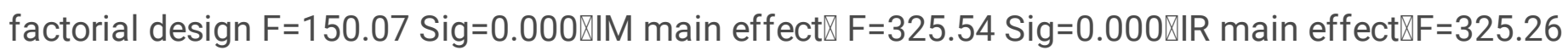

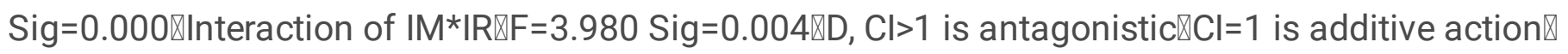

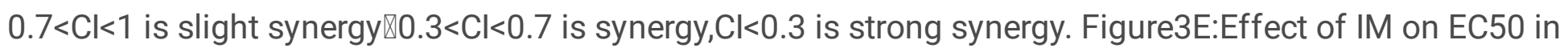
IR group,When IM is OuM, 1uM, 5uM and 10uM,the EC50 of IR group are 231.78Gy, 37.85Gy, 15.95Gy and

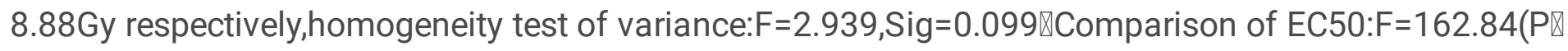


0.01) $₫ T$ Tamhane correction multiple comparison: $I M=0$ VS $I M=1: F=16.42(P \bigotimes 0.01), I M=1$ VS IM=5: $F=2.89(P$ $\nabla 0.01), I M=5$ VS $I M=10: F=2.53(p=0.29)$ $\mathbb{F}$ igure3F,G: The effects of $I M$ alone, IR group and combination group on the clonogenesis of GIST-882 cells:One way ANOVA,LED multiple comparison $₫ C$ tr VS $I M=1$ uM( $(P=0.018)$, Ctr VS IR=100Gy $(P=0.001)$, Ctr VS Combined group $(P=0.000) \otimes I M=1$ uM VS Combined group $(P=0.002), I R=100 G y$ VS Combined group $(P=0.28) \rrbracket$

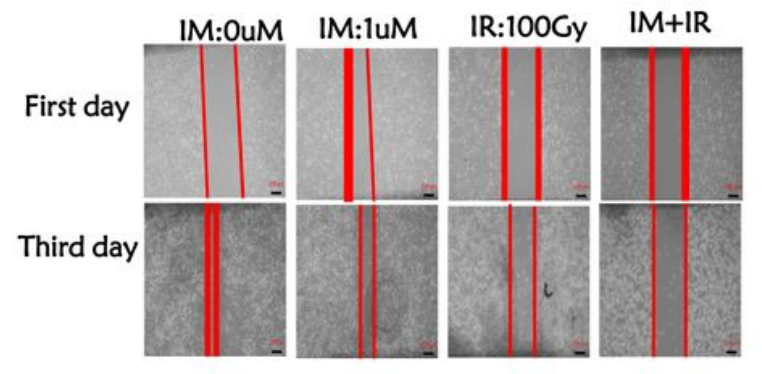

A

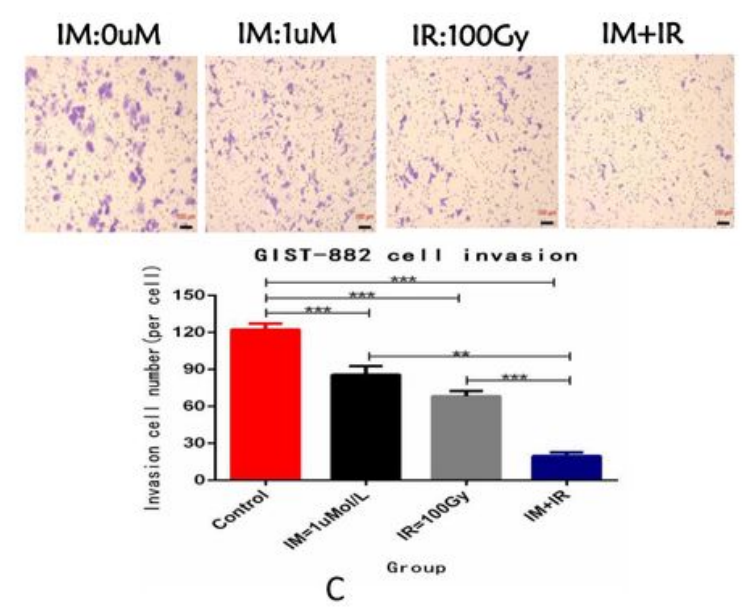

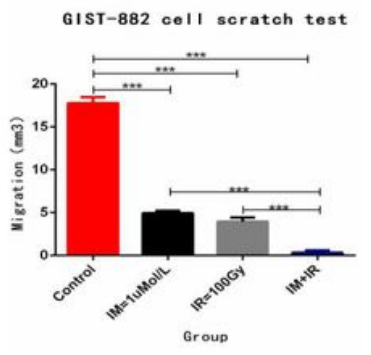

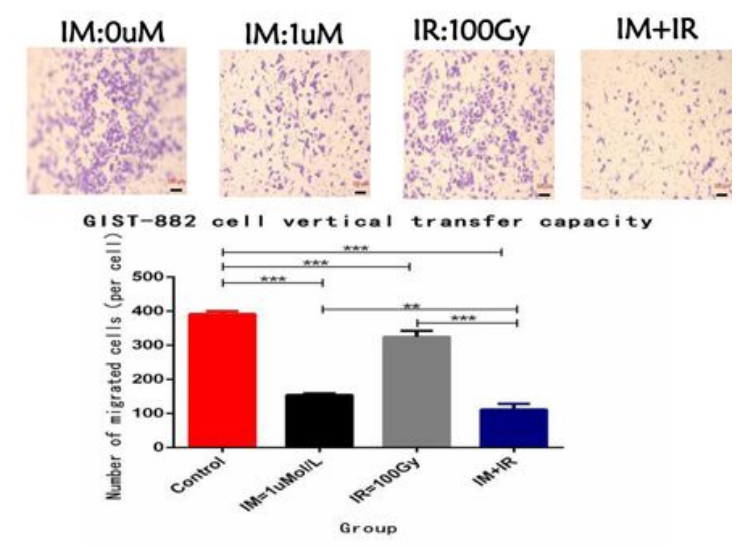

B

\section{Figure 4}

The migration and invasion of GIST-882 cells were influenced by IM alone, IR group and combination group;A,Scratch test: the difference of lateral migration area of GIST-882 cells between IM, IR and combination groups,Analysis of variance between groups: $F=507.71(P<0.01)$, LSD multiple

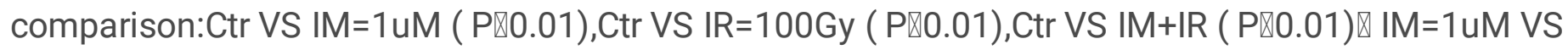
$I M+I R(P \otimes 0.01), I R=100 G y$ VS IM+IR(Pख0.01)『B,Longitudinal migration experiment:homogeneity test of variance: $F=1.17(p=0.38)$;Analysis of variance between groups: $F=323.88(P \otimes 0.01), L S D$ multiple

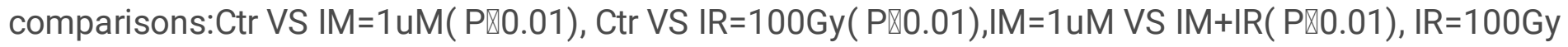
VS IM+IR( $P \otimes 0.01) ; C$,Invasive experiment:homogeneity test of variance: $F=0.945(p=0.463)$, analysis of variance between groups: $F=215.39$ ( $P \otimes 0.01$ ), LSD multiple comparisons: $C \operatorname{tr} V S I M=1 u M(P \otimes 0.01)$, Ctr VS $I R=100 G y(P \otimes 0.01), I M=14 M$ VS IM+IR( P『0.01), IR=100Gy VS IM+IR(Pख0.01); 

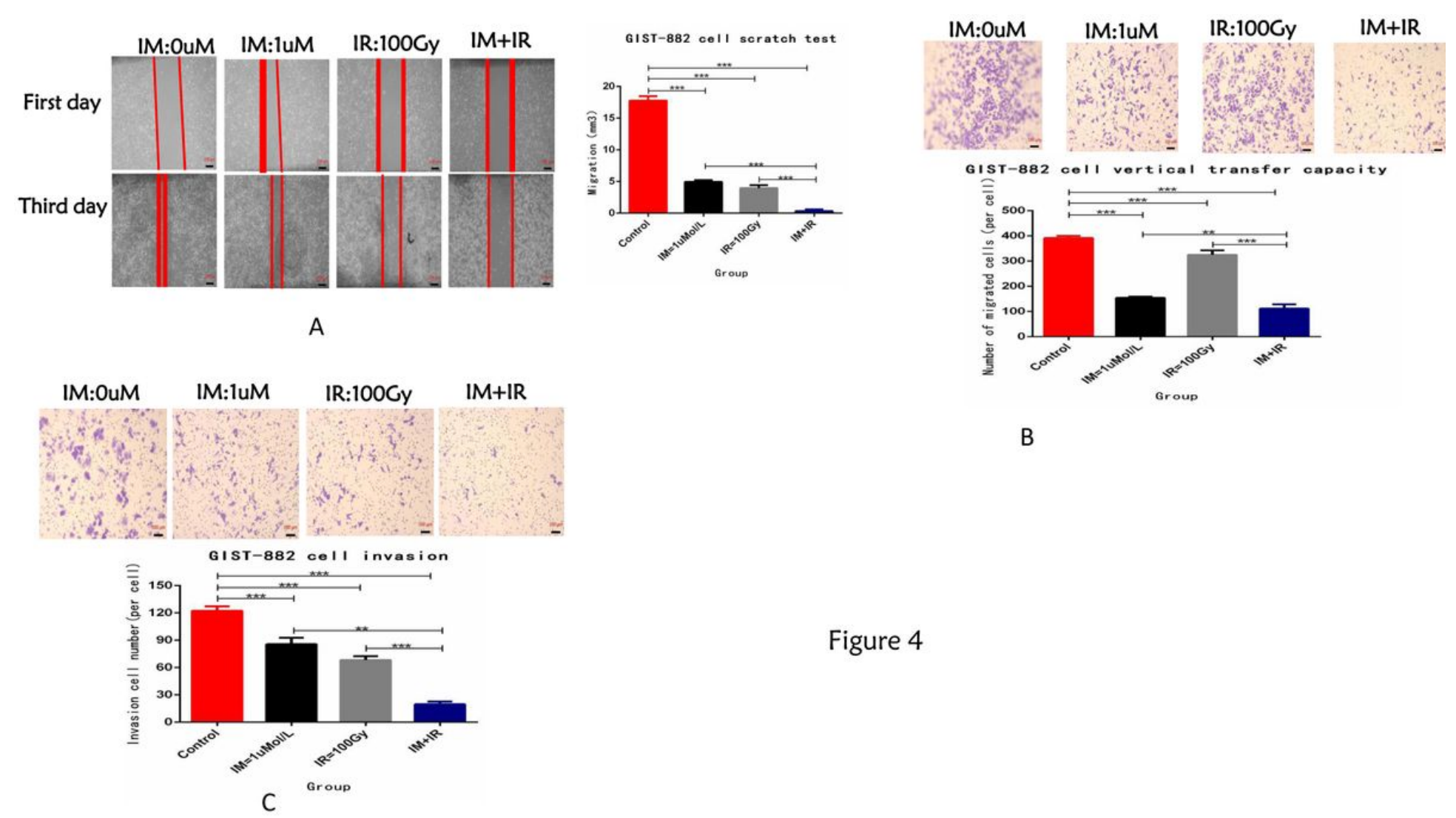

Figure 4

\section{Figure 4}

The migration and invasion of GIST-882 cells were influenced by IM alone, IR group and combination group;A,Scratch test: the difference of lateral migration area of GIST-882 cells between IM, IR and combination groups,Analysis of variance between groups: $F=507.71(P<0.01)$, LSD multiple

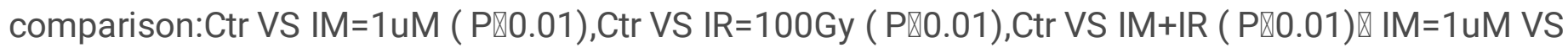
$I M+I R(P \otimes 0.01), I R=100 G y$ VS IM+IR(Pख0.01)षB,Longitudinal migration experiment:homogeneity test of variance: $F=1.17(p=0.38)$;Analysis of variance between groups: $F=323.88(P \llbracket 0.01), L S D$ multiple

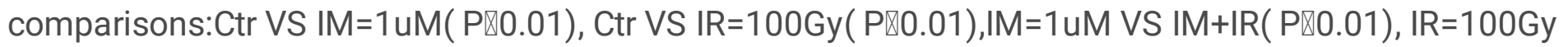
VS IM+IR( $P \otimes 0.01) ; C$,Invasive experiment:homogeneity test of variance: $F=0.945(p=0.463)$, analysis of variance between groups: $F=215.39$ ( $P \otimes 0.01)$, LSD multiple comparisons: $C \operatorname{tr} V S I M=1 u M(P \otimes 0.01)$, Ctr VS $I R=100 G y(P \otimes 0.01), I M=1$ uM VS IM+IR( $P \otimes 0.01), I R=100 G y$ VS IM+IR(P『0.01); 

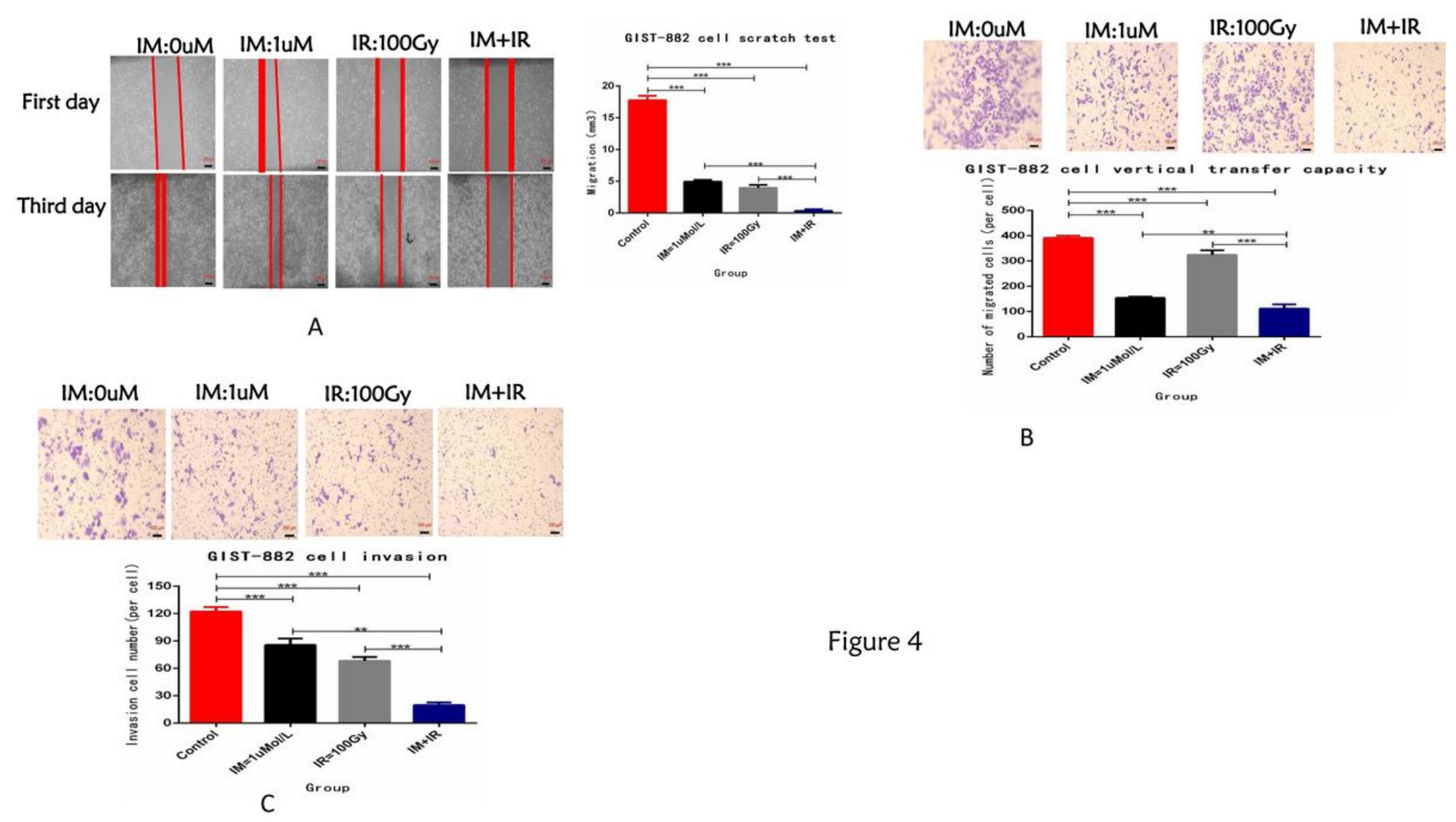

Figure 4

\section{Figure 4}

The migration and invasion of GIST-882 cells were influenced by IM alone, IR group and combination group;A,Scratch test: the difference of lateral migration area of GIST-882 cells between IM, IR and combination groups,Analysis of variance between groups: $F=507.71(P<0.01)$, LSD multiple

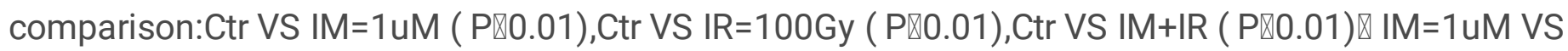
$I M+I R(P \otimes 0.01), I R=100 G y$ VS IM+IR(Pख0.01)षB,Longitudinal migration experiment:homogeneity test of variance: $F=1.17(p=0.38)$;Analysis of variance between groups: $F=323.88(P \otimes 0.01), L S D$ multiple

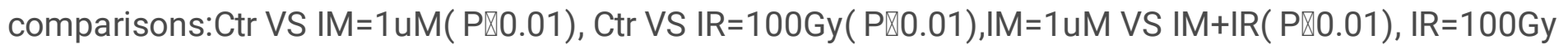
VS IM+IR( $P \llbracket 0.01) ; C$,Invasive experiment:homogeneity test of variance: $F=0.945(p=0.463)$, analysis of variance between groups:F=215.39( $P \otimes 0.01$ ), LSD multiple comparisons: $C t r$ VS IM=1uM( $P \otimes 0.01)$, Ctr VS $I R=100 G y(P \otimes 0.01), I M=14 M$ VS IM+IR(Pख0.01), IR=100Gy VS IM+IR(P冈0.01); 

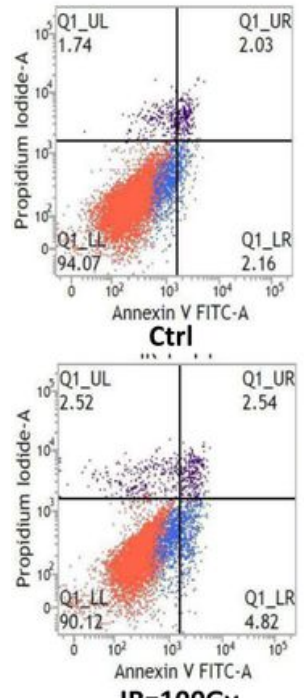

IR=100Gy
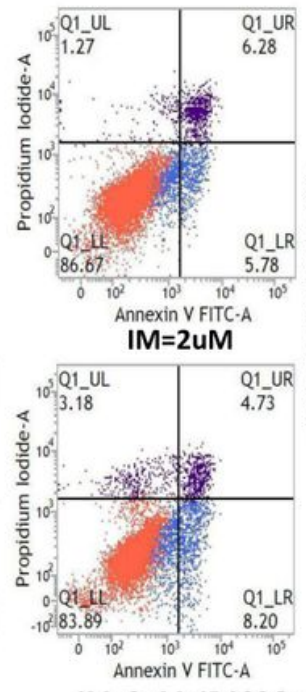

IM=2uM+IR100Gy
GIST-882 cell apoptosis

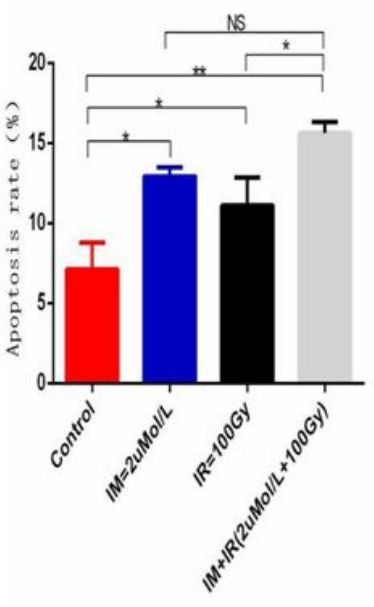

A
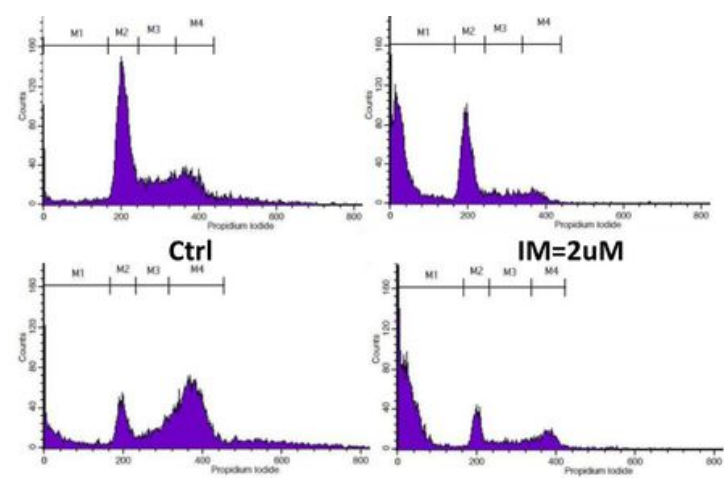

IM2uM+IR100Gy

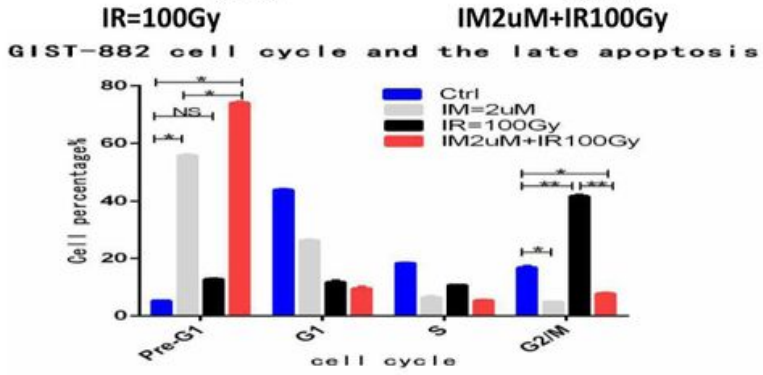

B

Figure 5

\section{Figure 5}

A.Statistics of apoptosis rate of GIST-882 cells,compared with the control group,the number of apoptosis cells in IM=2uMLIR=100Gy and IM2uM+IR100Gy increased significantly,the difference was statistically significant ( $p=0.011, p=0.035, p=0.003)$, and the combined group had the most apoptosis,but compared with $I M=2 u M$ group, there was no significant difference $(P=0.102)$,compared with $I R=100 G y$ group, the difference was statistically significant $(P=0.024)$;B,Statistics of cell cycle and late apoptosis of GIST882,In G0/G1 phase: compared with the control group,IM=2uM group could significantly enhance the rate of late apoptosis $(P<0.05)$, while IR = 100Gy group had no enhancement $(P>0.05)$;Compared with $I M=$ 2uM group, IM=2uM + IR=100Gy group significantly increased the rate of late apoptosis $(P<0.05) ; I n G 2 / M$ phase: compared with the control group,there was significant cell cycle arrest in IR $=100 \mathrm{~Gy}$ group $(P<0.05)$, but there was no cell cycle arrest in $I M=2 u M$ group and $I M=2 u M+I R=100 G y$ group $(P>0.05)$; 


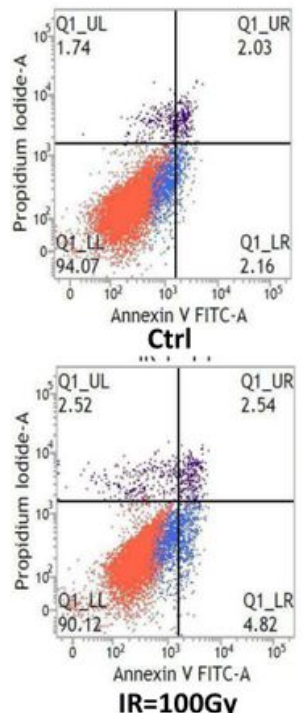

IR=100Gy
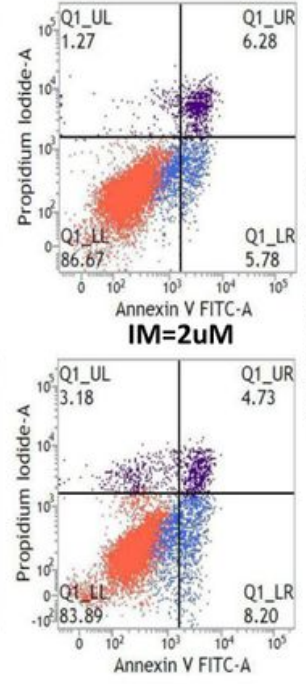

IM=2uM+IR100Gy
GIST-882 cell apoptosis

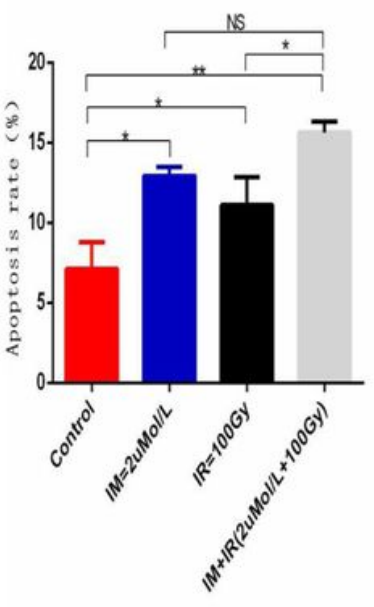

A
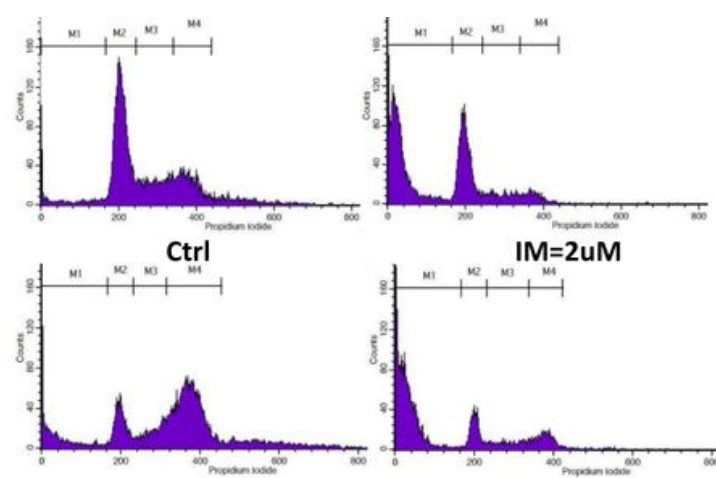

$\mathrm{IM}=2 \mathrm{uM}$

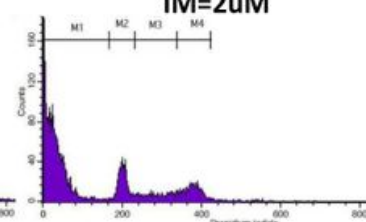

IM2uM+IR100Gy

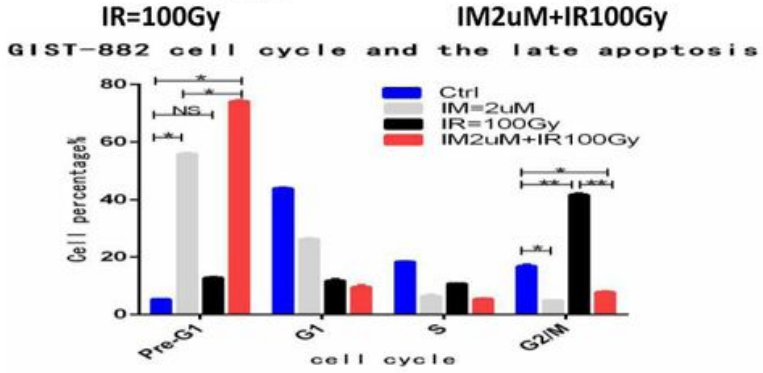

B

Figure 5

\section{Figure 5}

A.Statistics of apoptosis rate of GIST-882 cells,compared with the control group,the number of apoptosis cells in IM=2uM IR=100Gy and IM2uM+IR100Gy increased significantly,the difference was statistically significant $(p=0.011, p=0.035, p=0.003)$, and the combined group had the most apoptosis,but compared with $\mathrm{IM}=2 \mathrm{uM}$ group, there was no significant difference $(\mathrm{P}=0.102)$, compared with $\mathrm{IR}=100 \mathrm{~Gy}$ group, the difference was statistically significant $(P=0.024)$;B,Statistics of cell cycle and late apoptosis of GIST882,In G0/G1 phase: compared with the control group,IM=2uM group could significantly enhance the rate of late apoptosis $(P<0.05)$, while IR = 100Gy group had no enhancement $(P>0.05)$;Compared with IM = 2uM group, $I M=2 u M+I R=100 G y$ group significantly increased the rate of late apoptosis $(P<0.05) ; I n G 2 / M$ phase: compared with the control group,there was significant cell cycle arrest in IR = 100Gy group $(P<0.05)$, but there was no cell cycle arrest in $I M=2 u M$ group and $I M=2 u M+I R=100 G y$ group $(P>0.05)$; 


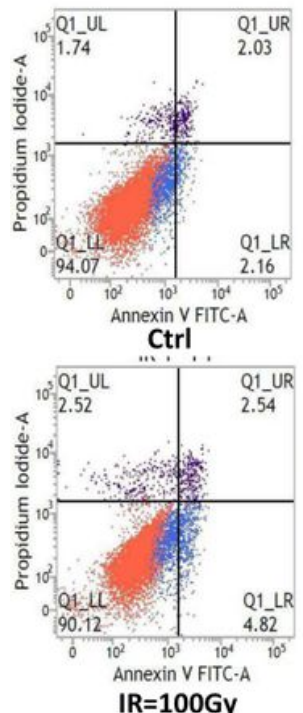

IR=100Gy
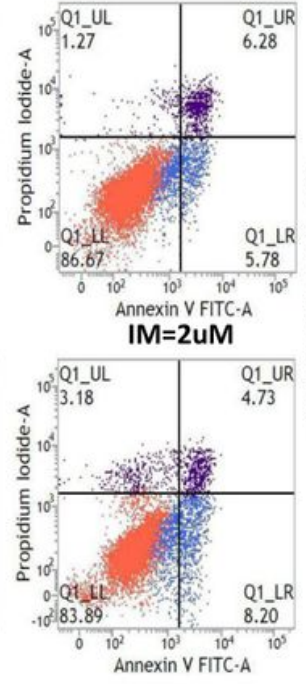

IM=2uM+IR100Gy
GIST-882 cell apoptosis

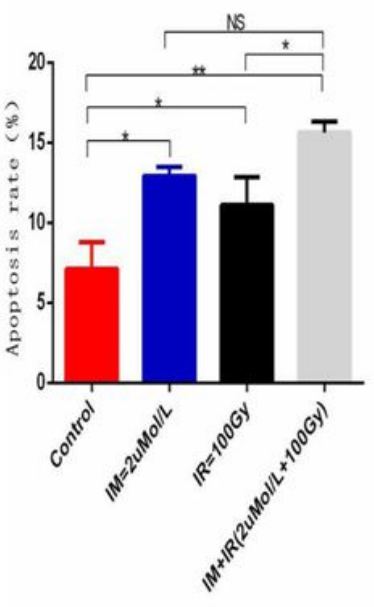

A
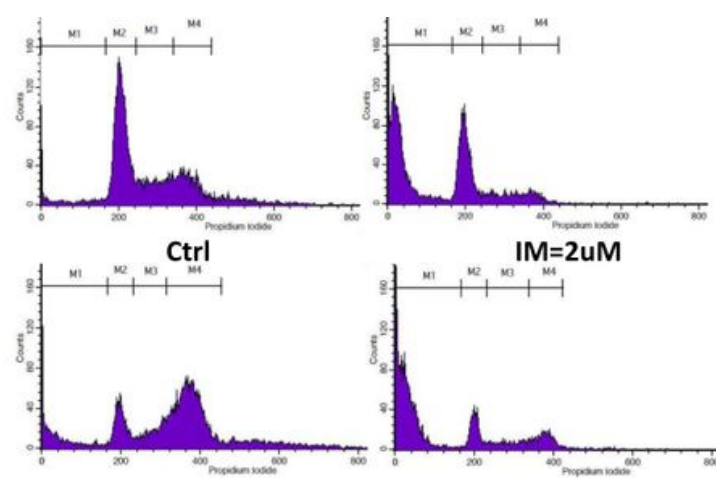

$\mathrm{IM}=2 \mathrm{uM}$

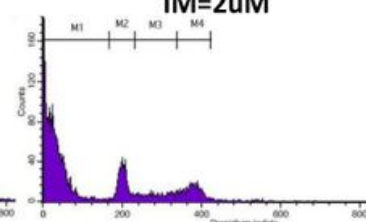

IM2uM+IR100Gy

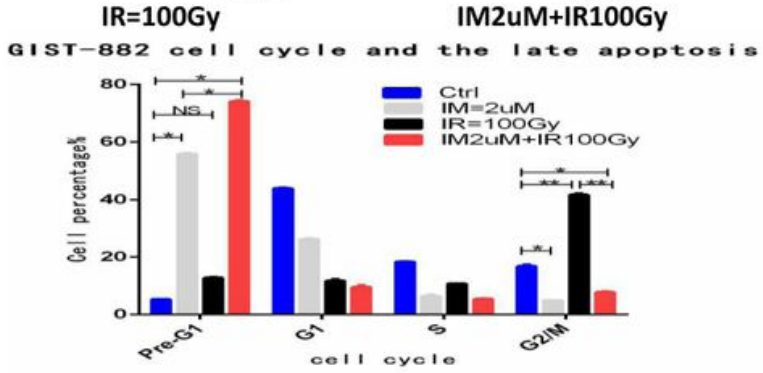

B

Figure 5

\section{Figure 5}

A.Statistics of apoptosis rate of GIST-882 cells,compared with the control group,the number of apoptosis cells in IM=2uM IR=100Gy and IM2uM+IR100Gy increased significantly,the difference was statistically significant $(p=0.011, p=0.035, p=0.003)$, and the combined group had the most apoptosis,but compared with $\mathrm{IM}=2 \mathrm{uM}$ group, there was no significant difference $(\mathrm{P}=0.102)$, compared with $\mathrm{IR}=100 \mathrm{~Gy}$ group, the difference was statistically significant $(P=0.024)$;B,Statistics of cell cycle and late apoptosis of GIST882,In G0/G1 phase: compared with the control group,IM=2uM group could significantly enhance the rate of late apoptosis $(\mathrm{P}<0.05)$, while IR = 100Gy group had no enhancement $(\mathrm{P}>0.05)$;Compared with IM = 2uM group, $I M=2 u M+I R=100 G y$ group significantly increased the rate of late apoptosis $(P<0.05) ; I n G 2 / M$ phase: compared with the control group,there was significant cell cycle arrest in IR = 100Gy group $(P<0.05)$, but there was no cell cycle arrest in $I M=2 u M$ group and $I M=2 u M+I R=100 G y$ group $(P>0.05)$; 

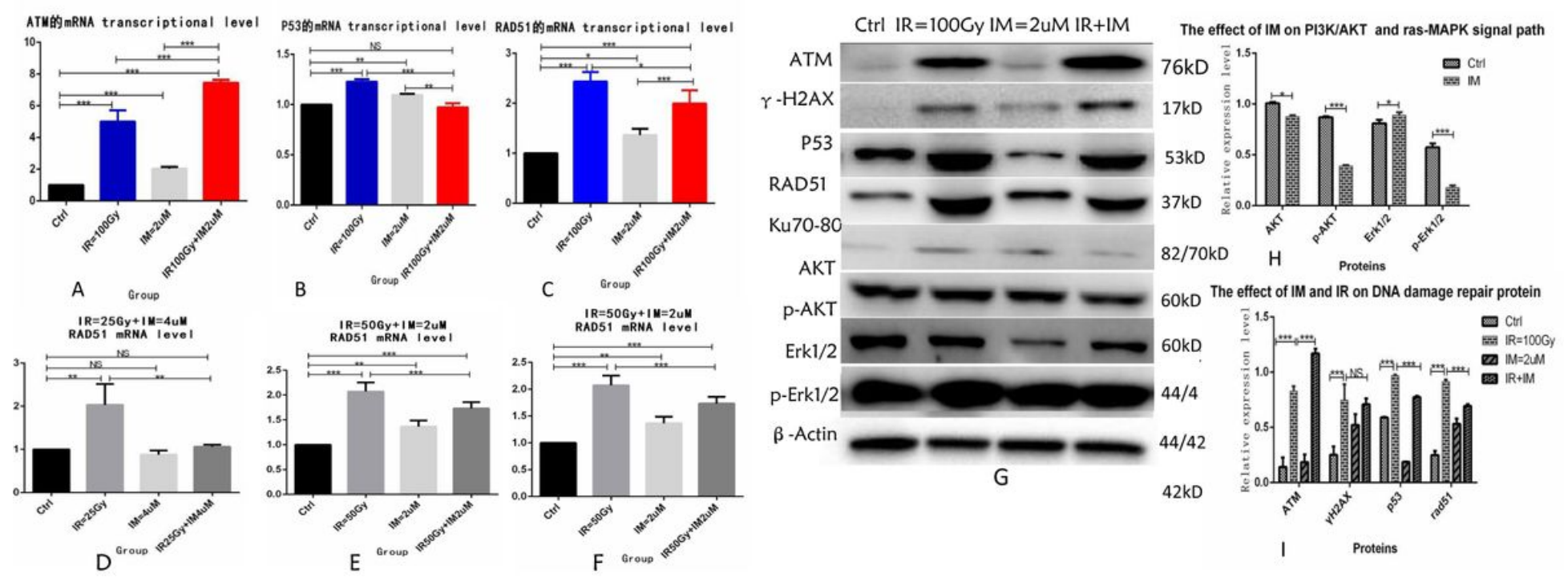

Figure 6

\section{Figure 6}

A. ATM(Ataxia telanggiectasia-mutated gene) :Compared with the control group,IM $\mathbb{I R}$ and IM $+\mathrm{IR}$ all can promote the transcription of ATM gene $(\mathrm{p} 1<0.01, \mathrm{p} 2<0.01$, $\mathrm{p} 3<0.01)$, and compared with IR group, the transcription of ATM gene in the combined group increased significantly $(\mathrm{p} 1<0.01)$;B. Compared with the control group IM and IR can promote the transcription of $p 53$ gene $(p 1<0.01, p 2<0.01)$, but there is no significant difference between the combined group and the control group, and compared with the IR group, the transcription of p53 in the combined group is significantly down regulated $(\mathrm{p} 1<0.01)$; $\mathrm{C}$. Compared with the control group, IM $\otimes$ IR and IM + IR can promote the transcription of Rad51 gene ( $1<0.05, \mathrm{p} 2<0.01, \mathrm{p} 3<0.01$ ), and the transcription of Rad51 in the combined group was significantly down regulated ( $\mathrm{p} 1<0.05)$; DaE and F:The transcription level of Rad51 was inhibited in diffwerent combination group compared with corresponding IR group ( $p<0.01, P<0.05, p<0.05)$, it is suggested that IM can inhibit the increase of transcription of Rad51 stimulated by IR, and thus inhibit DNA repair induced by ionizing radiation, so as to achieve radiation sensitization; $G$. The change of gene protein level in gist882 cells by im combined with IR: H:IM leads to the inhibition of Akt/PI3K and Ras-MAPK signaling pathways, especially the inhibition of Akt and ERK1/2 protein phosphorylation ( $\mathrm{p} 1<0.01$, p2<0.01);I,Compared with the control group, the expression of ATM, $\gamma^{-} \mathrm{H} 2 \mathrm{AX}, \mathrm{p} 53$ and Rad51 was increased by IR alone $(\mathrm{p} 1<0.01, \mathrm{p} 2<0.01, \mathrm{p} 3<0.01$, p4<0.01). Compared with IR alone, the expression of ATM protein was accumulated ( $1<0.01)$, while the expression of $\mathrm{p} 53$ and Rad51 was inhibited by combined action $(\mathrm{p} 1<0.01, \mathrm{p} 2<0.01)$; 

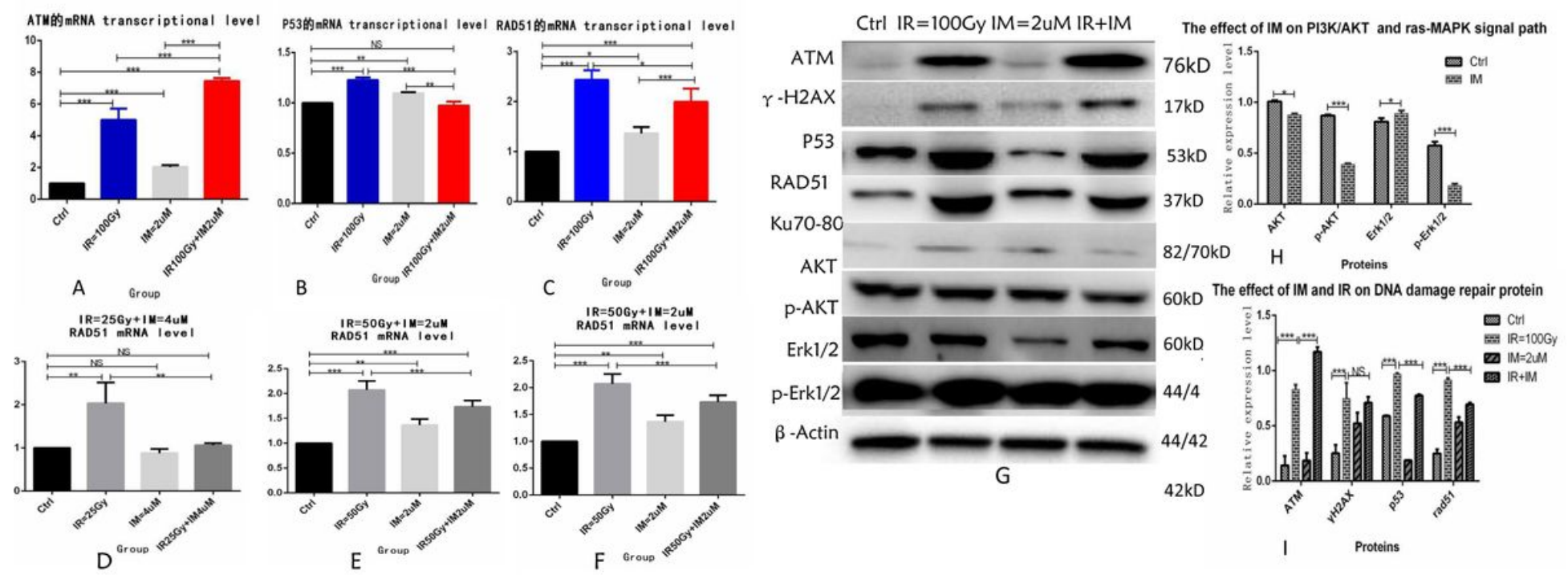

Figure 6

\section{Figure 6}

A. ATM(Ataxia telanggiectasia-mutated gene) :Compared with the control group,IM $\mathbb{I R}$ and IM $+\mathrm{IR}$ all can promote the transcription of ATM gene $(\mathrm{p} 1<0.01, \mathrm{p} 2<0.01$, $\mathrm{p} 3<0.01)$, and compared with IR group, the transcription of ATM gene in the combined group increased significantly $(\mathrm{p} 1<0.01)$;B. Compared with the control group IM and IR can promote the transcription of p53 gene $(p 1<0.01, p 2<0.01)$, but there is no significant difference between the combined group and the control group, and compared with the IR group, the transcription of p53 in the combined group is significantly down regulated $(\mathrm{p} 1<0.01)$; $\mathrm{C}$. Compared with the control group, IM $\otimes$ IR and IM + IR can promote the transcription of Rad51 gene ( $1<0.05, \mathrm{p} 2<0.01, \mathrm{p} 3<0.01$ ), and the transcription of Rad51 in the combined group was significantly down regulated ( $\mathrm{p} 1<0.05) ; \mathrm{D} \square \mathrm{E}$ and $\mathrm{F}$ :The transcription level of Rad51 was inhibited in diffwerent combination group compared with corresponding IR group ( $p<0.01, P<0.05, p<0.05)$, it is suggested that IM can inhibit the increase of transcription of Rad51 stimulated by IR, and thus inhibit DNA repair induced by ionizing radiation, so as to achieve radiation sensitization; $G$. The change of gene protein level in gist882 cells by im combined with IR: H:IM leads to the inhibition of Akt/PI3K and Ras-MAPK signaling pathways, especially the inhibition of Akt and ERK1/2 protein phosphorylation ( $\mathrm{p} 1<0.01$, p2<0.01);I,Compared with the control group, the expression of ATM, $\gamma^{-} \mathrm{H} 2 \mathrm{AX}, \mathrm{p} 53$ and Rad51 was increased by IR alone $(\mathrm{p} 1<0.01, \mathrm{p} 2<0.01, \mathrm{p} 3<0.01$, p4<0.01). Compared with IR alone, the expression of ATM protein was accumulated ( $1<0.01)$, while the expression of $\mathrm{p} 53$ and Rad51 was inhibited by combined action $(\mathrm{p} 1<0.01, \mathrm{p} 2<0.01)$; 

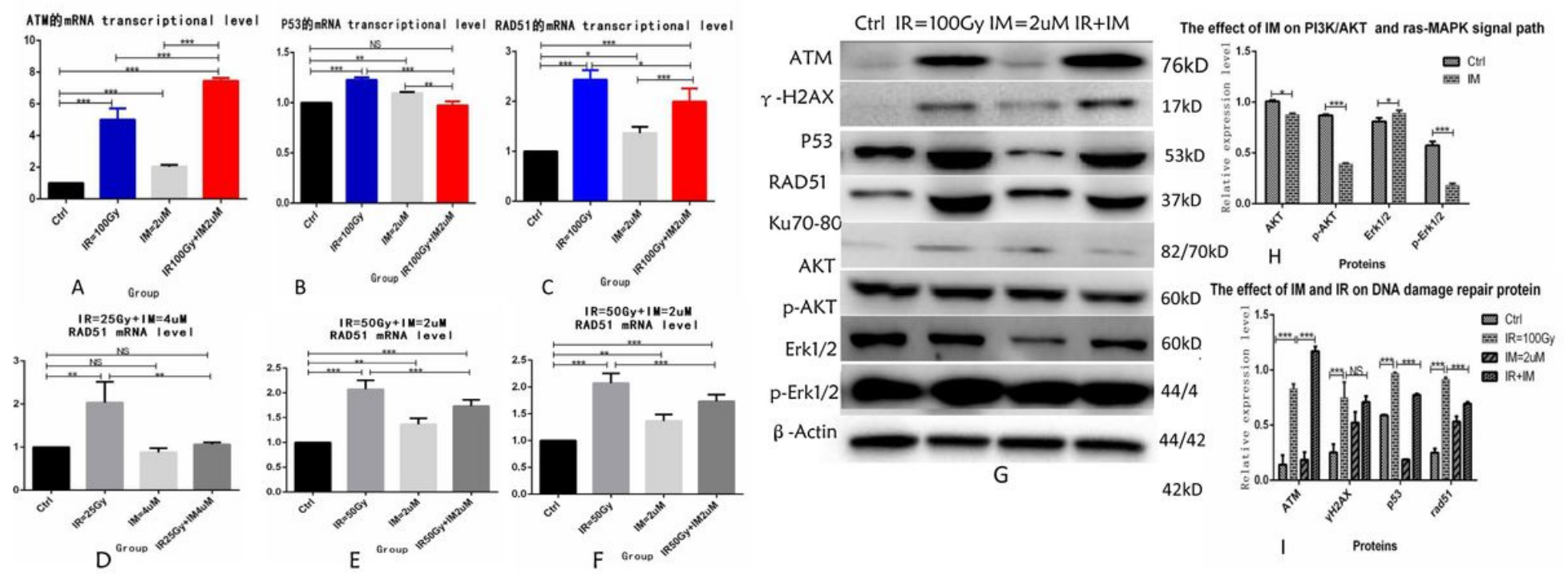

Figure 6

\section{Figure 6}

A. ATM(Ataxia telanggiectasia-mutated gene) :Compared with the control group,IM $\mathbb{I R}$ and IM $+\mathrm{IR}$ all can promote the transcription of ATM gene $(p 1<0.01, p 2<0.01, p 3<0.01)$, and compared with IR group, the transcription of ATM gene in the combined group increased significantly $(\mathrm{p} 1<0.01)$;B. Compared with the control group IM and IR can promote the transcription of p53 gene $(p 1<0.01, p 2<0.01)$, but there is no significant difference between the combined group and the control group, and compared with the IR group, the transcription of p53 in the combined group is significantly down regulated ( $1<0.01)$;C. Compared with the control group, IM $\otimes$ IR and IM + IR can promote the transcription of Rad51 gene ( $1<0.05, \mathrm{p} 2<0.01, \mathrm{p} 3<0.01$ ), and the transcription of Rad51 in the combined group was significantly down regulated ( $\mathrm{p} 1<0.05)$; DaE and F:The transcription level of Rad51 was inhibited in diffwerent combination group compared with corresponding IR group $(p<0.01, P<0.05, p<0.05)$, it is suggested that IM can inhibit the increase of transcription of Rad51 stimulated by IR, and thus inhibit DNA repair induced by ionizing radiation, so as to achieve radiation sensitization; $G$. The change of gene protein level in gist882 cells by im combined with IR: H:IM leads to the inhibition of Akt/PI3K and Ras-MAPK signaling pathways, especially the inhibition of Akt and ERK1/2 protein phosphorylation $(\mathrm{p} 1<0.01$, p2<0.01);I,Compared with the control group, the expression of ATM, $\gamma^{-} \mathrm{H} 2 \mathrm{AX}, \mathrm{p} 53$ and Rad51 was increased by IR alone $(p 1<0.01, p 2<0.01, p 3<0.01$, p4<0.01). Compared with IR alone, the expression of ATM protein was accumulated ( $1<0.01$ ), while the expression of $\mathrm{p} 53$ and Rad51 was inhibited by combined action ( $1<0.01, \mathrm{p} 2<0.01)$;

\section{Supplementary Files}

This is a list of supplementary files associated with this preprint. Click to download.

- Additionalfile1.docx 
- Additionalfile1.docx

- Additionalfile1.docx

- Additionalfile2.docx

- Additionalfile2.docx

- Additionalfile2.docx

- Additionalfile3.docx

- Additionalfile3.docx

- Additionalfile3.docx 